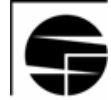

II ASA

The Pontryagin Maximum Principle and Transversality Conditions for a Class of Optimal Control Problems with Infinite Time Horizons

Sergei M. Aseev and Arkady V. Kryazhimskiy

RP-05-003

June 2005

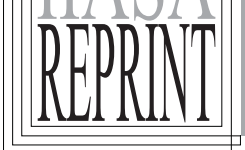





\section{The Pontryagin Maximum Principle and Transversality Conditions for a Class of Optimal Control Problems with Infinite Time Horizons}

Sergei M. Aseev

Arkady V. Kryazhimskiy

RP-05-003

June 2005

Reprinted from SIAM Journal on Control and Optimization, 43(3):1094-1119

International Institute for Applied Systems Analysis - Schlossplatz 1 - A-2361 Laxenburg • Austria Tel: (+43 2236) 807 • Fax: (+43 2236) 71313 •E-mail: publications@iiasa.ac.at•Web: www.iiasa.ac.at 
IIASA Reprints make research conducted at the International Institute for Applied Systems Analysis more accessible to a wider audience. They reprint independently reviewed articles that have been previously published in journals. Views or opinions expressed herein do not necessarily represent those of the Institute, its National Member Organizations, or other organizations supporting the work.

Reprinted with permission from SIAM Journal on Control and Optimization, 43(3):1094-1119. Copyright @ 2004 Society for Industrial and Applied Mathematics.

All rights reserved. No part of this publication may be reproduced or transmitted in any form or by any means, electronic or mechanical, including photocopy, recording, or any information storage or retrieval system, without permission in writing from the copyright holder. 


\title{
THE PONTRYAGIN MAXIMUM PRINCIPLE AND TRANSVERSALITY CONDITIONS FOR A CLASS OF OPTIMAL CONTROL PROBLEMS WITH INFINITE TIME HORIZONS*
}

\author{
SERGEI M. ASEEV ${ }^{\dagger}$ AND ARKADY V. KRYAZHIMSKIY ${ }^{\dagger}$
}

\begin{abstract}
This paper suggests some further developments in the theory of first-order necessary optimality conditions for problems of optimal control with infinite time horizons. We describe an approximation technique involving auxiliary finite-horizon optimal control problems and use it to prove new versions of the Pontryagin maximum principle. Special attention is paid to the behavior of the adjoint variables and the Hamiltonian. Typical cases, in which standard transversality conditions hold at infinity, are described. Several significant earlier results are generalized.
\end{abstract}

Key words. optimal control, infinite horizon, Pontryagin maximum principle, transversality conditions, optimal economic growth

AMS subject classifications. 49K15, 91B62

DOI. $10.1137 / \mathrm{S} 0363012903427518$

1. Introduction. We deal with the following infinite-horizon optimal control problem $(\mathrm{P})$ :

$$
\begin{gathered}
\dot{x}(t)=f(x(t), u(t)), \quad u(t) \in U ; \\
x(0)=x_{0} ; \\
\text { maximize } J(x, u)=\int_{0}^{\infty} e^{-\rho t} g(x(t), u(t)) d t .
\end{gathered}
$$

Here $x(t)=\left(x^{1}(t), \ldots, x^{n}(t)\right) \in \mathbb{R}^{n}$ and $u(t)=\left(u^{1}(t), \ldots, u^{m}(t)\right) \in \mathbb{R}^{m}$ are the current values of the system's states and controls; $U$ is a nonempty convex compactum in $\mathbb{R}^{m} ; x_{0}$ is a given initial state; and $\rho \geq 0$ is a discount parameter. The functions $f: G \times U \mapsto \mathbb{R}^{n}, g: G \times U \mapsto \mathbb{R}^{1}$, the matrix $\partial f / \partial x=\left(\partial f^{i} / \partial x^{j}\right)_{i, j=1, \ldots, n}$, and the gradient $\partial g / \partial x=\left(\partial g / \partial x^{1}, \ldots, \partial g / \partial x^{n}\right)$ are assumed to be continuous on $G \times U$. Here $G$ is an open set in $\mathbb{R}^{n}$ such that $x_{0} \in G$. As usual an admissible control in system (1.1) is identified with an arbitrary measurable function $u:[0, \infty) \mapsto U$. A trajectory corresponding to a control $u$ is a Carathéodory solution $x$ to (1.1), which satisfies the initial condition (1.2). We assume that, for any control $u$, a trajectory $x$ corresponding to $u$ exists on $[0, \infty)$ and takes values in $G$ (due to the continuous differentiability of $f$, the trajectory $x$ is unique). Any pair $(u, x)$, where $u$ is a control and $x$ the trajectory corresponding to $u$, will be called an admissible pair.

Problems of this type naturally arise in the studies on optimization of economic growth (see [1], [2], [14], [23], [27], [33], [39]). Progress in this field of economics was initiated by Ramsey in the 1920s [35].

* Received by the editors May 12, 2003; accepted for publication (in revised form) February 20, 2004; published electronically November 9, 2004. This work was supported by the Fujitsu Research Institute (IIASA-FRI contract 01-109).

http://www.siam.org/journals/sicon/43-3/42751.html

${ }^{\dagger}$ International Institute for Applied Systems Analysis, Schlossplatz 1, Laxenburg, A-2361, Austria and Steklov Institute of Mathematics, Gubkina str. 8, Moscow, 119991, Russia (aseev@iiasa.ac.at, aseev@mi.ras.ru; kryazhim@mtu-net.ru). The first author was partially supported by the Russian Foundation for Basic Research (project 99-01-01051). The second author was partially supported by the Russian Foundation for Basic Research (project 03-01-00737). 
Our basic assumptions are the following.

(A1) There exists a $C \geq 0$ such that

$$
\langle x, f(x, u)\rangle \leq C\left(1+\|x\|^{2}\right) \text { for all } \quad x \in G \text { and all } u \in U .
$$

(A2) For each $x \in G$, the function $u \mapsto f(x, u)$ is affine, i.e.,

$$
f(x, u)=f_{0}(x)+\sum_{i=1}^{m} f_{i}(x) u^{i} \quad \text { for all } \quad x \in G \quad \text { and all } \quad u \in U,
$$

where $f_{i}: G \mapsto \mathbb{R}^{n}, i=0,1, \ldots, m$, are continuously differentiable.

(A3) For each $x \in G$, the function $u \mapsto g(x, u)$ is concave.

(A4) There exist positive-valued functions $\mu$ and $\omega$ on $[0, \infty)$ such that $\mu(t) \rightarrow 0$, $\omega(t) \rightarrow 0$ as $t \rightarrow \infty$, and for any admissible pair $(u, x)$,

$$
\begin{gathered}
e^{-\rho t} \max _{u \in U}|g(x(t), u)| \leq \mu(t) \quad \text { for all } \quad t>0 ; \\
\int_{T}^{\infty} e^{-\rho t}|g(x(t), u(t))| d t \leq \omega(T) \quad \text { for all } \quad T>0 .
\end{gathered}
$$

Assumption (A1) is conventionally used in existence theorems in the theory of optimal control (see [19], [22]). Assumptions (A2) and (A3) imply that problem (P) is "linear-convex" in control; the "linear-convex" structure is important for the implementation of approximation techniques. The second condition in (A4) implies that the integral (1.3) converges absolutely for any admissible pair $(u, x)$, which excludes any ambiguity in interpreting problem (P). As shown in [13, Theorem 3.6], assumptions (A1)-(A4) guarantee the existence of an admissible optimal pair in problem (P).

In this paper, we develop first-order necessary optimality conditions for problem $(\mathrm{P})$. Note that, for infinite-horizon optimal control problems without a discounting factor $(\rho=0)$, the Pontryagin maximum principle was stated in [34]. For problems involving a positive discounting factor $(\rho>0)$, a general statement on the Pontryagin maximum principle was given in [24]. However, both statements establish the "core" relations of the Pontryagin maximum principle only and do not suggest any analogue of the transversality conditions, which constitute an immanent component of the Pontryagin maximum principle for classical finite-horizon optimal control problems with nonconstrained terminal states. The issue of transversality conditions for problem $(\mathrm{P})$ is the focus of our study.

Introduce the Hamilton-Pontryagin function $\mathcal{H}: G \times[0, \infty) \times U \times \mathbb{R}^{n} \times \mathbb{R}^{1} \mapsto \mathbb{R}^{1}$ and the Hamiltonian $H: G \times[0, \infty) \times \mathbb{R}^{n} \times \mathbb{R}^{1} \mapsto \mathbb{R}^{1}$ for problem $(\mathrm{P})$ :

$$
\begin{aligned}
\mathcal{H}\left(x, t, u, \psi, \psi^{0}\right) & =\langle f(x, u), \psi\rangle+\psi^{0} e^{-\rho t} g(x, u) ; \\
H\left(x, t, \psi, \psi^{0}\right) & =\sup _{u \in U} \mathcal{H}\left(x, t, u, \psi, \psi^{0}\right) .
\end{aligned}
$$

The Pontryagin maximum principle involves an admissible pair $\left(u_{*}, x_{*}\right)$ and a pair $\left(\psi, \psi^{0}\right)$ of adjoint variables associated with $\left(u_{*}, x_{*}\right)$; here $\psi$ is a solution to the adjoint equation

$$
\dot{\psi}(t)=-\left[\frac{\partial f\left(x_{*}(t), u_{*}(t)\right)}{\partial x}\right]^{*} \psi(t)-\psi^{0} e^{-\rho t} \frac{\partial g\left(x_{*}(t), u_{*}(t)\right)}{\partial x}
$$


on $[0, \infty)$, and $\psi^{0}$ is a nonnegative real; $\left(\psi, \psi^{0}\right)$ is said to be nontrivial if

$$
\|\psi(0)\|+\psi^{0}>0 .
$$

We shall use the following definition. We shall say that an admissible pair $\left(u_{*}, x_{*}\right)$ satisfies the core Pontryagin maximum principle (in problem $(\mathrm{P})$ ), together with a pair $\left(\psi, \psi^{0}\right)$ of adjoint variables associated with $\left(u_{*}, x_{*}\right)$, if $\left(\psi, \psi^{0}\right)$ is nontrivial and the following maximum condition holds:

$$
\mathcal{H}\left(x_{*}(t), t, u_{*}(t), \psi(t), \psi^{0}\right)=H\left(x_{*}(t), t, \psi(t), \psi^{0}\right) \quad \text { for a.a. } \quad t \geq 0 .
$$

Of special interest is the case where problem $(\mathrm{P})$ is not abnormal, i.e., when the Lagrange multiplier $\psi^{0}$ in the core Pontryagin maximum principle does not vanish. In this case we do not lose generality if we set $\psi^{0}=1$. Accordingly, we define the normal-form Hamilton-Pontryagin function $\tilde{\mathcal{H}}: G \times[0, \infty) \times U \times \mathbb{R}^{n} \mapsto \mathbb{R}^{1}$ and the normal-form Hamiltonian $\tilde{H}: G \times[0, \infty) \times \mathbb{R}^{n} \mapsto \mathbb{R}^{1}$ as follows:

$$
\begin{aligned}
\tilde{\mathcal{H}}(x, t, u, \psi) & =\mathcal{H}(x, t, u, \psi, 1)=\langle f(x, u), \psi\rangle+e^{-\rho t} g(x, u) ; \\
\tilde{H}(x, t, \psi) & =H(x, t, \psi, 1)=\sup _{u \in U} \tilde{\mathcal{H}}(x, t, u, \psi) .
\end{aligned}
$$

Given an admissible pair $\left(u_{*}, x_{*}\right)$, introduce the normal-form adjoint equation

$$
\dot{\psi}(t)=-\left[\frac{\partial f\left(x_{*}(t), u_{*}(t)\right)}{\partial x}\right]^{*} \psi(t)-e^{-\rho t} \frac{\partial g\left(x_{*}(t), u_{*}(t)\right)}{\partial x} .
$$

Any solution $\psi$ to $(1.7)$ on $[0, \infty)$ will be called an adjoint variable associated with $\left(u_{*}, x_{*}\right)$. We shall say that an admissible pair $\left(u_{*}, x_{*}\right)$ satisfies the normal-form core Pontryagin maximum principle together with an adjoint variable $\psi$ associated with $\left(u_{*}, x_{*}\right)$ if the following normal-form maximum condition holds:

$$
\tilde{\mathcal{H}}\left(x_{*}(t), t, u_{*}(t), \psi(t)\right)=\tilde{H}\left(x_{*}(t), t, \psi(t)\right) \quad \text { for a.a. } \quad t \geq 0 .
$$

In the context of problem $(\mathrm{P}),[24]$ states the following (see also [17]).

THEOREM 1. If an admissible pair $\left(u_{*}, x_{*}\right)$ is optimal in problem $(\mathrm{P})$, then $\left(u_{*}, x_{*}\right)$ satisfies relations (1.4)-(1.6) of the core Pontryagin maximum principle together with some pair $\left(\psi, \psi^{0}\right)$ of adjoint variables associated with $\left(u_{*}, x_{*}\right)$.

Qualitatively, this formulation is weaker than the corresponding statement known for finite-horizon optimal control problems with nonconstrained terminal states. Indeed, consider the following finite-horizon counterpart of problem $(\mathrm{P})$.

Problem $\left(\mathrm{P}_{T}\right)$ :

$$
\begin{gathered}
\dot{x}(t)=f(x(t), u(t)), \quad u(t) \in U ; \\
x(0)=x_{0} \\
\text { maximize } J_{T}(x, u)=\int_{0}^{T} e^{-\rho t} g(x(t), u(t)) d t
\end{gathered}
$$

here $T>0$ is a fixed positive real. The classical theory [34] says that if an admissible pair $\left(u_{*}, x_{*}\right)$ is optimal in problem $\left(\mathrm{P}_{T}\right)$, then $\left(u_{*}, x_{*}\right)$ satisfies the core Pontryagin 
maximum principle together with some pair $\left(\psi, \psi^{0}\right)$ of adjoint variables associated with $\left(u_{*}, x_{*}\right)$, and, moreover, $\left(\psi, \psi^{0}\right)$ satisfies the transversality conditions

$$
\psi^{0}=1, \quad \psi(T)=0 .
$$

In Theorem 1 any analogue of the transversality conditions (1.9) is missing.

There were numerous attempts to find specific situations in which the infinitehorizon Pontryagin maximum principle holds together with additional boundary conditions at infinity (see [12], [15], [16], [21], [26], [31], [36], [38]). However, the major results were established under rather severe assumptions of linearity or full convexity, which made it difficult to apply them to particular meaningful problems (see, e.g., [28] discussing the application of the Pontryagin maximum principle to a particular infinite-horizon optimal control problem).

In this paper we follow the approximation approach suggested in [9], [10], and [11]. We approximate problem $(\mathrm{P})$ by a sequence of finite-horizon optimal control problems $\left\{\left(\mathrm{P}_{k}\right)\right\}(k=1,2, \ldots)$ whose horizons go to infinity. Problems $\left(\mathrm{P}_{k}\right)(k=1,2, \ldots)$ impose no constraints on the terminal states; in this sense, they inherit the structure of problem $(\mathrm{P})$; on the other hand, problems $\left(\mathrm{P}_{k}\right)$ are not plain "restrictions" of problem $(\mathrm{P})$ to finite intervals like problem $\left(\mathrm{P}_{T}\right)$ : the goal functionals in problems $\left(\mathrm{P}_{k}\right)$ include special penalty terms associated with a certain control optimal in problem $(\mathrm{P})$. This approach allows us to find limit forms of the classical transversality conditions for problems $\left(\mathrm{P}_{k}\right)$ as $k \rightarrow \infty$ and formulate conditions that complement the core Pontryagin maximum principle and hold with a necessity for every admissible pair optimal in problem (P). The results presented here generalize [9], [10], [11], and [12].

Earlier, a similar approximation approach was used to derive necessary optimality conditions for various nonclassical optimal control problems (see, e.g., [3], [4], [5], [7], [32], and also survey [6]). Based on relevant approximation techniques and the methodology presented here, one can extend the results of this paper to more complex infinite-horizon problems of optimal control (e.g., problems with nonsmooth data). In this paper, our primary goal is to show how the approximation approach allows us to resolve the major singularity emerging due to the unboundedness of the time horizon. Therefore, we restrict our consideration to the relatively simple nonlinear infinitehorizon problem $(\mathrm{P})$, which is smooth, "linear-convex" in control, and free from any constraints on the system's states.

Finally, we note that the suggested approximation methodology, appropriately modified, can be used directly in analysis of particular nonstandard optimal control problems with infinite time horizons (see, e.g., [8]).

2. Transversality conditions: Counterexamples. Considering problem $(\mathrm{P})$ as the "limit" of finite-horizon problems $\left(\mathrm{P}_{T}\right)$ whose horizons $T$ tend to infinity, one can expect the following "natural" transversality conditions for problem $(\mathrm{P})$ :

$$
\psi^{0}=1, \quad \lim _{t \rightarrow \infty} \psi(t)=0 ;
$$

here $\left(\psi, \psi^{0}\right)$ is a pair of adjoint variables satisfying the core Pontryagin maximum principle together with an admissible pair $\left(u_{*}, x_{*}\right)$ optimal in problem $(\mathrm{P})$. The relations

$$
\psi^{0}=1, \quad \lim _{t \rightarrow \infty}\left\langle\psi(t), x_{*}(t)\right\rangle=0
$$

represent alternative transversality conditions for problem $(\mathrm{P})$, which are frequently used in economic applications (see, e.g., [14]). 
The interpretation of (2.2) as transversality conditions for problem (P) is also motivated by Arrow's statement on sufficient conditions of optimality (see [1], [2], and [36]), which (under some additional assumptions) asserts that if (2.2) holds for an admissible pair $\left(u_{*}, x_{*}\right)$ and a pair $\left(\psi, \psi^{0}\right)$ of adjoint variables, jointly satisfying the core Pontryagin maximum principle, then $\left(u_{*}, x_{*}\right)$ is optimal in problem $(\mathrm{P})$, provided the superposition $H\left(x, t, \psi(t), \psi^{0}\right)$ is concave in $x$. Another type of transversality condition formulated in terms of stability theory was proposed in [38]. In [12], global behavior of the adjoint variable associated with an optimal admissible pair was characterized in terms of appropriate integral functionals. In this paper, we concentrate on the derivation of pointwise transversality conditions of types (2.1) and $(2.2)$.

Note that, generally, for infinite-horizon optimal control problems neither transversality condition (2.1) nor (2.2) is valid. For the case of no discounting $(\rho=0)$, illustrating counterexamples were given in [24] and [37], and for problems with discounting $(\rho>0)$, some examples were given in [12] and [31]. In particular, [31] presents an example showing that an infinite-horizon optimal control problem with a positive discount can be abnormal; i.e., in the core Pontryagin maximum principle, the Lagrange multiplier $\psi^{0}$ may necessarily vanish (which contradicts both (2.1) and (2.2)).

Here, we provide further counterexamples for problem $(\mathrm{P})$ in the case where discount parameter $\rho$ is positive.

Example 1 shows that for problem $(\mathrm{P})$, the limit relation in (2.1) may be violated, whereas the alternative transversality conditions (2.2) may hold.

Example 1. Consider the optimal control problem

$$
\begin{gathered}
\dot{x}(t)=u(t)-x(t), \quad u(t) \in U=[0,1] ; \\
x(0)=\frac{1}{2} ; \\
\text { maximize } J(x, u)=\int_{0}^{\infty} e^{-t} \ln \frac{1}{x(t)} d t .
\end{gathered}
$$

We set $G=(0, \infty)$ and treat the above problem as problem (P). Assumptions (A1)(A4) are, obviously, satisfied. For an arbitrary trajectory $x$, we have $e^{-t} / 2 \leq x(t)<1$ for all $t \geq 0$. Hence, $\left(u_{*}, x_{*}\right)$, where $u_{*}(t) \stackrel{\text { a.e. }}{=} 0$ and $x_{*}(t)=e^{-t} / 2$ for all $t \geq 0$, is the unique optimal admissible pair. The Hamilton-Pontryagin function is given by

$$
\mathcal{H}\left(x, t, u, \psi, \psi^{0}\right)=(u-x) \psi-\psi^{0} e^{-t} \ln x .
$$

Let $\left(\psi, \psi^{0}\right)$ be an arbitrary pair of adjoint variables such that $\left(u_{*}, x_{*}\right)$ satisfies the core Pontryagin maximum principle together with $\left(\psi, \psi^{0}\right)$. The adjoint equation (1.4) has the form

$$
\dot{\psi}(t)=\psi(t)+\psi^{0} e^{-t} \frac{1}{x_{*}(t)}=\psi+2 \psi^{0},
$$

and the maximum condition (1.6) implies

$$
\psi(t) \leq 0 \text { for all } t \geq 0 .
$$

Assume $\psi^{0}=0$. Then $\psi(0)<0$ and $\psi(t)=e^{t} \psi(0) \rightarrow-\infty$ as $t \rightarrow \infty$; i.e., the limit relation in (2.1) does not hold. Let $\psi^{0}>0$. Without loss of generality (or multiplying 
both $\psi$ and $\psi^{0}$ by $\left.1 / \psi^{0}\right)$, we assume $\psi^{0}=1$. Then $\psi(t)=(\psi(0)+2) e^{t}-2$. By (2.3), only two cases are admissible: (a) $\psi(0)=-2$ and (b) $\psi(0)<-2$. In case (a) $\psi(t) \equiv-2$, and in case (b) $\psi(t) \rightarrow-\infty$ as $t \rightarrow \infty$. In both situations the limit relation in (2.1) is violated. Note that $\psi(t) \equiv-2(t \geq 0)$ and $\psi^{0}=1$ satisfy (2.2).

The next example is complementary to Example 1; it shows that for problem $(\mathrm{P})$, the limit relation in (2.2) may be violated, whereas (2.1) may hold.

Example 2. Consider the following optimal control problem:

$$
\begin{gathered}
\dot{x}(t)=u(t), \quad u(t) \in U=\left[\frac{1}{2}, 1\right] \\
x(0)=0 \\
\text { maximize } J(x, u)=\int_{0}^{\infty} e^{-t}(1+\gamma(x(t))) u(t) d t .
\end{gathered}
$$

Here $\gamma$ is a nonnegative continuously differentiable real function such that

$$
I=\int_{0}^{\infty} e^{-t} \gamma(t) d t<\infty
$$

We set $G=\mathbb{R}^{1}$. Clearly, assumptions (A1)-(A3) are satisfied. Below, we specify the form of $\gamma$ and show that assumption (A4) is satisfied too.

The admissible pair $\left(u_{*}, x_{*}\right)$, where $u_{*}(t) \stackrel{\text { a.e. }}{=} 1$ and $x_{*}(t)=t$ for all $t \geq 0$, is optimal. Indeed, let $(u, x)$ be an arbitrary admissible pair. Observing (2.4), we find that $\dot{x}(t)>0$ for a.a. $t \geq 0$. Taking $\tau(t)=x(t)$ for a new integration variable in (2.5), we get $d \tau=u(t) d t$ and

$$
t(\tau)=\int_{0}^{\tau} \frac{1}{u(t(s))} d s \text { for all } \tau \geq 0
$$

As far as

$$
\int_{0}^{\tau} \frac{1}{u(t(s))} d s \geq \tau
$$

we get

$$
\begin{aligned}
J(x, u) & =\int_{0}^{\infty} e^{-t}(1+\gamma(x(t))) u(t) d t=\int_{0}^{\infty} e^{-\int_{0}^{\tau} \frac{1}{u(t(s))} d s}(1+\gamma(\tau)) d \tau \\
& \leq \int_{0}^{\infty} e^{-\tau}(1+\gamma(\tau)) d \tau=J\left(u_{*}, x_{*}\right) .
\end{aligned}
$$

Hence, $\left(u_{*}, x_{*}\right)$ is an optimal admissible pair. It is easy to see that there are no other optimal admissible pairs. The Hamilton-Pontryagin function has the form

$$
\mathcal{H}\left(x, t, u, \psi, \psi^{0}\right)=u \psi+\psi^{0} e^{-t}(1+\gamma(x)) u .
$$

Let $\left(\psi, \psi^{0}\right)$ be an arbitrary pair of adjoint variables such that $\left(u_{*}, x_{*}\right)$ satisfies the core Pontryagin maximum principle together with $\left(\psi, \psi^{0}\right)$. The adjoint equation (1.4) has the form

$$
\dot{\psi}(t)=-\psi^{0} \dot{\gamma}(t) e^{-t}
$$


If $\psi^{0}=0$, then the maximum condition (1.6) implies $\psi(t) \equiv \psi(0)>0$; hence, $\psi(t) x_{*}(t)=\psi(0) t \rightarrow \infty$ as $t \rightarrow \infty$, and the limit relation in (2.2) is violated.

Suppose $\psi^{0}>0$, or, equivalently, $\psi^{0}=1$. Then, due to (1.4), we have

$$
\psi(t)=\psi(0)-\int_{0}^{t} \dot{\gamma}(s) e^{-s} d s .
$$

The limit relation in (2.2) has the form $\lim _{t \rightarrow \infty} t \psi(t)=0$. Let us show that one can define $\gamma$ so that the latter relation is violated; i.e., for any $\psi(0) \in \mathbb{R}^{1}$,

$$
p(t) \not 0 \text { as } t \rightarrow \infty,
$$

where $p(t)=t \psi(t)$. We represent $p(t)$ as follows:

$$
\begin{aligned}
p(t) & =t \psi(0)-t \int_{0}^{t} \dot{\gamma}(s) e^{-s} d s=t \psi(0)-t\left[\left.\gamma(s) e^{-s}\right|_{0} ^{t}+\int_{0}^{t} \gamma(s) e^{-s} d s\right] \\
& =t \psi(0)-t \gamma(t) e^{-t}+t \gamma(0)-t I(t),
\end{aligned}
$$

where

$$
I(t)=\int_{0}^{t} \gamma(s) e^{-s} d s
$$

Introducing $\nu(t)=\gamma(t) e^{-t}$, rewrite

$$
\begin{aligned}
& I(t)=\int_{0}^{t} \nu(s) d s \\
& p(t)=t \psi(0)-t \nu(t)+t \nu(0)-t I(t) .
\end{aligned}
$$

Due to $(2.6)$,

$$
\lim _{t \rightarrow \infty} I(t)=I .
$$

Now let us specify the form of $\nu$. For each natural $k$, we fix a positive $\varepsilon_{k}<1 / 2$ and denote by $\Delta_{k}$ the $\varepsilon_{k}$-neighborhood of $k$. Clearly, $\Delta_{k} \cup \Delta_{j}=\emptyset$ for $k \neq j$. We set

$$
\begin{gathered}
\nu(k)=\frac{1}{k} \quad \text { for } \quad k=1,2, \ldots ; \\
\nu(t)=0 \quad \text { for } t \notin \cup_{k=1}^{\infty} \Delta_{k} ; \\
\nu(t) \in\left[0, \frac{1}{k}\right] \quad \text { for } t \in \Delta_{k} \quad(k=1,2, \ldots) .
\end{gathered}
$$

Moreover, we require that

$$
\sum_{k=j}^{\infty} \int_{\Delta_{k}} \nu(t) d t \leq \frac{1}{j^{2}}
$$

This can be achieved, for example, by letting $\frac{2 \varepsilon_{k}}{k} \leq \frac{a_{k}}{k^{2}}$, where $\sum_{k=1}^{\infty} a_{k}=1, a_{k}>0$. Indeed, in this case

$$
\sum_{k=j}^{\infty} \int_{\Delta_{k}} \nu(t) d t \leq \sum_{k=j}^{\infty} \frac{2 \varepsilon_{k}}{k} \leq \sum_{k=j}^{\infty} \frac{a_{k}}{k^{2}} \leq \frac{1}{j^{2}} \sum_{k=j}^{\infty} a_{k} \leq \frac{1}{j^{2}}
$$


i.e., (2.11) holds. Note that, for $j=1$, the left-hand side in (2.11) equals $I$ (see (2.6)); thus, (2.11) implies that assumption (2.6) holds.

Another fact following from (2.11) is that

$$
\lim _{t \rightarrow \infty} t(I-I(t))=0 .
$$

Indeed, by (2.8), $I\left(j+\varepsilon_{j}\right)=\sum_{k=1}^{j} \int_{\Delta_{k}} \nu(t) d t$; hence, due to $(2.11)$,

$$
I-I\left(j+\varepsilon_{j}\right)=\sum_{k=j+1}^{\infty} \int_{\Delta_{k}} \nu(t) d t \leq \frac{1}{(j+1)^{2}} .
$$

For $t \in\left[j+\varepsilon_{j}, j+1+\varepsilon_{j+1}\right]$, we have $I\left(j+\varepsilon_{j}\right) \leq I(t) \leq I$; therefore, for $t \geq 1$,

$$
0 \leq I-I(t) \leq \frac{1}{(j+1)^{2}} \leq \frac{1}{\left(t-\varepsilon_{j+1}\right)^{2}} \leq \frac{1}{(t-1 / 2)^{2}},
$$

which yields (2.12). The given definition of $\nu$ is equivalent to defining $\gamma$ by

$$
\begin{gathered}
\gamma(k)=\frac{e^{k}}{k} \quad \text { for } \quad k=1,2, \ldots ; \\
\gamma(t)=0 \quad \text { for } t \notin \cup_{k=1}^{\infty} \Delta_{k} ; \\
\gamma(t) \in\left[0, \frac{e^{k}}{k}\right] \quad \text { for } \quad t \in \Delta_{k} \quad(k=1,2, \ldots)
\end{gathered}
$$

and requiring (2.11). Let us show that assumption (A4) is satisfied. Let $(u, x)$ be an arbitrary admissible pair. By $(2.4), t / 2 \leq x(t) \leq t$ for all $t \geq 0$. Hence, by the definition of $\nu$, we have $\nu(x(t)) \leq\left(\frac{t}{2}-1\right)^{-1}=\frac{2}{(t-2)}$ for all $t>2$. Hence,

$$
0 \leq e^{-\rho t} \max _{u \in U}\left[(1+\gamma(x(t)) u] \leq \mu(t)=e^{-\rho t}+\frac{2}{(t-2)} \rightarrow 0 \quad \text { as } \quad t \rightarrow \infty .\right.
$$

Thus, the first condition in (A4) holds. Furthermore, introducing the integration variable $\tau(t)=x(t)$, we get

$$
\begin{aligned}
\int_{T}^{\infty} e^{-t}(1+\gamma(x(t))) u(t) d t & =\int_{x(T)}^{\infty} e^{-\int_{0}^{\tau} \frac{1}{u(t(s))} d s}(1+\gamma(\tau)) d \tau \\
& \leq \int_{x(T)}^{\infty} e^{-\tau}(1+\gamma(\tau)) d \tau \leq \omega(T) \\
& =\int_{\frac{T}{2}}^{\infty} e^{-t}(1+\gamma(t)) d t \rightarrow 0 \quad \text { as } \quad T \rightarrow \infty
\end{aligned}
$$

Hence, the second condition in (A4) holds. We stated the validity of assumption (A4).

By the definition of $\gamma$, for $t \in \Delta_{k}, k=1,2, \ldots$, we have

$$
0 \leq t \nu(t) \leq \frac{k+\varepsilon_{k}}{k} \leq 1+\frac{1}{k} .
$$

Hence,

$$
0 \leq t \nu(t) \leq 2 \quad \text { for all } \quad t \geq 0
$$


i.e., the function $t \nu(t)$ is bounded. Furthermore, $k \nu(k)=1$, and due to (2.13) for any sequence $t_{k} \rightarrow \infty$ such that $t_{k} \in[k, k+1] \backslash\left(\Delta_{k} \cup \Delta_{k+1}\right)$, we have $t_{k} \nu\left(t_{k}\right)=0$. Therefore, $\lim _{t \rightarrow \infty} t \nu(t)$ does not exist.

Using $\nu(0)=0$, we specify $(2.9)$ as

$$
p(t)=t \psi(0)-t \nu(t)-t I(t) .
$$

If $\psi(0)>I$, then, in view of $(2.10), \lim _{t \rightarrow \infty} t(\psi(0)+I(t))=\infty$, which implies $\lim _{t \rightarrow \infty} p(t)=\infty$, since $t \nu(t)$ is bounded. Similarly, we find that if $\psi(0)<I$, then $\lim _{t \rightarrow \infty} p(t)=-\infty$. Let, finally, $\psi(0)=I$. Then,

$$
\lim _{t \rightarrow \infty} t(\psi(0)-I(t))=\lim _{t \rightarrow \infty} t(I-I(t))=0,
$$

as follows from (2.12). Thus, in the right-hand side of (2.15) the sum of the first and third terms has the zero limit at infinity, whereas the second term, $t \nu(t)$, has no limit at infinity, as we noted earlier. Consequently, $p(t)$, the left-hand side in (2.15), has no limit at infinity. We showed that $(2.7)$ holds for every $\psi(0) \in \mathbb{R}^{1}$.

Thus, the limit relation in the transversality conditions (2.2) is violated. Note that setting $\psi^{0}=1$ and $\psi(0)=I$, we make the adjoint variable $\psi$ satisfy the transversality conditions (2.1). Indeed, in this case $\psi(t)=p(t) / t=\psi(0)-I-\nu(t)$ for all $t>0$, and the conditions $\psi(0)=I$ and (2.14) imply that $\psi(t) \rightarrow 0$ as $t \rightarrow \infty$.

Examples 1 and 2 show that assumptions (A1)-(A4) are insufficient for the validity of the core Pontryagin maximum principle together with the transversality conditions (2.1) or (2.2) as necessary conditions of optimality in problem (P). Below, we find mild additional assumptions that guarantee that necessary conditions of optimality in problem $(\mathrm{P})$ include the core Pontryagin maximum principle and transversality conditions of type (2.1) or of type (2.2).

3. Basic constructions. In this section, we define a sequence of finite-horizon optimal control problems $\left\{\left(\mathrm{P}_{k}\right)\right\}(k=1,2, \ldots)$ with horizons $T_{k} \rightarrow \infty$; we treat problems $\left(\mathrm{P}_{k}\right)$ as approximations to the infinite-horizon problem $(\mathrm{P})$.

Let us describe the data defining problems $\left(\mathrm{P}_{k}\right)(k=1,2, \ldots)$. Given a control $u_{*}$ optimal in problem $(\mathrm{P})$, we fix a sequence of continuously differentiable functions $z_{k}:[0, \infty) \rightarrow \mathbb{R}^{m}(k=1,2, \ldots)$ and a sequence of positive $\sigma_{k}(k=1,2, \ldots)$ such that

$$
\begin{gathered}
\sup _{t \in[0, \infty)}\left\|z_{k}(t)\right\| \leq \max _{u \in U}\|u\|+1 ; \\
\int_{0}^{\infty} e^{-(\rho+1) t}\left\|z_{k}(t)-u_{*}(t)\right\|^{2} d t \leq \frac{1}{k} ; \\
\sup _{t \in[0, \infty)}\left\|\dot{z}_{k}(t)\right\| \leq \sigma_{k}<\infty ; \\
\sigma_{k} \rightarrow \infty \text { as } \quad k \rightarrow \infty
\end{gathered}
$$

(obviously, such sequences exist). Next, we take a monotonically increasing sequence of positive $T_{k}$ such that $T_{k} \rightarrow \infty$ as $k \rightarrow \infty$ and

$$
\omega\left(T_{k}\right) \leq \frac{1}{k\left(1+\sigma_{k}\right)} \quad \text { for all } \quad k=1,2, \ldots ;
$$

recall that $\omega$ is defined in (A4). For every $k=1,2, \ldots$, we define problem $\left(\mathrm{P}_{k}\right)$ as follows. 
Problem $\left(\mathrm{P}_{k}\right)$ :

$$
\begin{gathered}
\dot{x}(t)=f(x(t), u(t)), \quad u(t) \in U ; \\
x(0)=x_{0} ;
\end{gathered}
$$

$\operatorname{maximize} J_{k}(x, u)=\int_{0}^{T_{k}} e^{-\rho t} g(x(t), u(t)) d t-\frac{1}{1+\sigma_{k}} \int_{0}^{T_{k}} e^{-(\rho+1) t}\left\|u(t)-z_{k}(t)\right\|^{2} d t$.

By Theorem 9.3.i of [19], for every $k=1,2, \ldots$ there exists an admissible pair $\left(u_{k}, x_{k}\right)$ optimal in problem $\left(\mathrm{P}_{k}\right)$.

The above-defined sequence of problems, $\left\{\left(\mathrm{P}_{k}\right)\right\}(k=1,2, \ldots)$, will be said to be associated with the control $u_{*}$.

We are ready to formulate our basic approximation lemma.

Lemma 1. Let assumptions (A1)-(A4) be satisfied; let $u_{*}$ be a control optimal in problem $(\mathrm{P})$; let $\left\{\left(\mathrm{P}_{k}\right)\right\}(k=1,2, \ldots)$ be the sequence of problems associated with $u_{*}$; and for every $k=1,2, \ldots$, let $u_{k}$ be a control optimal in problem $\left(\mathrm{P}_{k}\right)$. Then, for every $T>0$, it holds that $u_{k} \rightarrow u_{*}$ in $L^{2}\left([0, T], \mathbb{R}^{m}\right)$ as $k \rightarrow \infty$.

Proof. Take a $T>0$. Let $k_{1}$ be such that $T_{k_{1}} \geq T$. For every $k \geq k_{1}$, we have

$$
\begin{aligned}
J_{k}\left(x_{k}, u_{k}\right) & =\int_{0}^{T_{k}} e^{-\rho t}\left[g\left(x_{k}(t), u_{k}(t)\right)-e^{-t} \frac{\left\|u_{k}(t)-z_{k}(t)\right\|^{2}}{1+\sigma_{k}}\right] d t \\
& \leq \int_{0}^{T_{k}} e^{-\rho t} g\left(x_{k}(t), u_{k}(t)\right) d t-\frac{e^{-(\rho+1) T}}{1+\sigma_{k}} \int_{0}^{T}\left\|u_{k}(t)-z_{k}(t)\right\|^{2} d t
\end{aligned}
$$

where $x_{k}$ is the trajectory corresponding to $u_{k}$. Hence, introducing the trajectory $x_{*}$ corresponding to $u_{*}$ and taking into account the optimality of $u_{k}$ in problem $\left(\mathrm{P}_{k}\right)$, optimality of $u_{*}$ in problem (P), assumption (A4), and conditions (3.2) and (3.4), we find that, for all sufficiently large $k$,

$$
\begin{aligned}
\frac{e^{-(\rho+1) T}}{1+\sigma_{k}} \int_{0}^{T}\left\|u_{k}(t)-z_{k}(t)\right\|^{2} d t \leq & \int_{0}^{T_{k}} e^{-\rho t} g\left(x_{k}(t), u_{k}(t)\right) d t-J_{k}\left(x_{*}, u_{*}\right) \\
\leq & \int_{0}^{T_{k}} e^{-\rho t} g\left(x_{k}(t), u_{k}(t)\right) d t-J\left(x_{*}, u_{*}\right) \\
& +\omega\left(T_{k}\right)+\int_{0}^{\infty} \frac{e^{-(\rho+1) t}}{1+\sigma_{k}}\left\|u_{*}(t)-z_{k}(t)\right\|^{2} d t \\
\leq & \int_{0}^{T_{k}} e^{-\rho t} g\left(x_{k}(t), u_{k}(t)\right) d t-J\left(x_{*}, u_{*}\right)+\frac{2}{k\left(1+\sigma_{k}\right)} \\
\leq & J\left(x_{k}, u_{k}\right)-J\left(x_{*}, u_{*}\right)+\frac{3}{k\left(1+\sigma_{k}\right)} \leq \frac{3}{k\left(1+\sigma_{k}\right)} .
\end{aligned}
$$

Hence,

$$
\left\|u_{k}-z_{k}\right\|_{L^{2}}^{2} \leq \frac{3 e^{(\rho+1) T}}{k}
$$


Then, in view of (3.2),

$$
\begin{aligned}
\left\|u_{k}-u_{*}\right\|_{L^{2}} & \leq\left(\int_{0}^{T}\left\|u_{*}(t)-z_{k}(t)\right\|^{2} d t\right)^{1 / 2}+\left(\int_{0}^{T}\left\|u_{k}(t)-z_{k}(t)\right\|^{2} d t\right)^{1 / 2} \\
& \leq\left(\frac{e^{(\rho+1) T}}{k}\right)^{1 / 2}+\left(\frac{3 e^{(\rho+1) T}}{k}\right)^{1 / 2}=(1+\sqrt{3})\left(\frac{e^{(\rho+1) T}}{k}\right)^{1 / 2} .
\end{aligned}
$$

Therefore, for any $\epsilon>0$, there exists a $k_{2} \geq k_{1}$ such that $\left\|u_{k}-u_{*}\right\|_{L^{2}} \leq \epsilon$ for all $k \geq k_{2}$.

Now, based on Lemma 1, we derive a limit form of the classical Pontryagin maximum principle for problems $\left(\mathrm{P}_{k}\right)(k=1,2, \ldots)$, which leads us to the core Pontryagin maximum principle for problem $(\mathrm{P})$.

We use the following formulation of the Pontryagin maximum principle [34] for problems $\left(\mathrm{P}_{k}\right)(k=1,2, \ldots)$. Let an admissible pair $\left(u_{k}, x_{k}\right)$ be optimal in problem $\left(\mathrm{P}_{k}\right)$ for some $k$. Then there exists a pair $\left(\psi_{k}, \psi_{k}^{0}\right)$ of adjoint variables associated with $\left(u_{k}, x_{k}\right)$ such that $\left(u_{k}, x_{k}\right)$ satisfies relations (1.4)-(1.6) of the core Pontryagin maximum principle (in problem $\left.\left(\mathrm{P}_{k}\right)\right)$ together with $\left(\psi_{k}, \psi_{k}^{0}\right)$ and, moreover, $\psi_{k}^{0}>0$ and the transversality condition

$$
\psi_{k}\left(T_{k}\right)=0
$$

holds; recall that $\psi_{k}$ is a solution on $\left[0, T_{k}\right]$ to the adjoint equation associated with $\left(u_{k}, x_{k}\right)$ in problem $\left(\mathrm{P}_{k}\right)$, i.e.,

$$
\dot{\psi}_{k}(t) \stackrel{\text { a.e. }}{=}-\left[\frac{\partial f\left(x_{k}(t), u_{k}(t)\right)}{\partial x}\right]^{*} \psi_{k}(t)-\psi^{0} e^{-\rho t} \frac{\partial g\left(x_{k}(t), u_{k}(t)\right)}{\partial x},
$$

and the core Pontryagin maximum principle satisfied by $\left(u_{k}, x_{k}\right)$, together with $\left(\psi_{k}, \psi_{k}^{0}\right)$, implies that the following maximum condition holds:

$$
\mathcal{H}_{k}\left(x_{k}(t), t, u_{k}(t), \psi_{k}(t), \psi_{k}^{0}\right) \stackrel{\text { a.e. }}{=} H_{k}\left(x_{k}(t), t, \psi_{k}(t), \psi_{k}^{0}\right) ;
$$

here $\mathcal{H}_{k}$ and $H_{k}$, given by

$$
\begin{aligned}
\mathcal{H}_{k}\left(x, t, u, \psi, \psi^{0}\right) & =\langle f(x, u), \psi\rangle+\psi^{0} e^{-\rho t} g(x, u)-\psi^{0} e^{-(\rho+1) t} \frac{\left\|u-z_{k}(t)\right\|^{2}}{1+\sigma_{k}} \\
H_{k}\left(x, t, \psi, \psi^{0}\right) & =\sup _{u \in U} \mathcal{H}_{k}\left(x, t, u, \psi, \psi^{0}\right)
\end{aligned}
$$

are, respectively, the Hamilton-Pontryagin function and the Hamiltonian in problem $\left(\mathrm{P}_{k}\right)$; note that in [34] it is shown that (3.6) and (3.7) imply

$$
\frac{d}{d t} H_{k}\left(x_{k}(t), t, \psi_{k}(t), \psi_{k}^{0}\right) \stackrel{\text { a.e. }}{=} \frac{\partial \mathcal{H}_{k}}{\partial t}\left(x_{k}(t), t, u_{k}(t), \psi_{k}(t), \psi_{k}^{0}\right) .
$$

Lemma 2. Let assumptions (A1)-(A4) be satisfied; let $\left(u_{*}, x_{*}\right)$ be an admissible pair optimal in problem $(\mathrm{P})$; let $\left\{\left(\mathrm{P}_{k}\right)\right\}(k=1,2, \ldots)$ be the sequence of problems associated with $u_{*}$; for every $k=1,2, \ldots$, let $\left(u_{k}, x_{k}\right)$ be an admissible pair optimal in problem $\left(\mathrm{P}_{k}\right)$; for every $k=1,2, \ldots$, let $\left(\psi_{k}, \psi_{k}^{0}\right)$ be a pair of adjoint variables associated with $\left(u_{k}, x_{k}\right)$ in problem $\left(\mathrm{P}_{k}\right)$ such that $\left(u_{k}, x_{k}\right)$ satisfies relations (3.6) 
and (3.7) of the core Pontryagin maximum principle in problem $\left(\mathrm{P}_{k}\right)$ together with $\left(\psi_{k}, \psi_{k}^{0}\right)$; and for every $k=1,2, \ldots$, one has $\psi_{k}^{0}>0$, and the transversality condition (3.5) holds. Finally, let the sequences $\left\{\psi_{k}(0)\right\}$ and $\left\{\psi_{k}^{0}\right\}$ be bounded and

$$
\left\|\psi_{k}(0)\right\|+\psi_{k}^{0} \geq a \quad(k=1,2, \ldots)
$$

for some $a>0$. Then there exists a subsequence of $\left\{\left(u_{k}, x_{k}, \psi_{k}, \psi_{k}^{0}\right)\right\}$, denoted again as $\left\{\left(u_{k}, x_{k}, \psi_{k}, \psi_{k}^{0}\right)\right\}$, such that

(i) for every $T>0$,

$$
\begin{gathered}
u_{k}(t) \rightarrow u_{*}(t) \quad \text { for a.a. } t \in[0, T] \text { as } k \rightarrow \infty \\
x_{k} \rightarrow x_{*} \quad \text { uniformly on }[0, T] \text { as } k \rightarrow \infty
\end{gathered}
$$

$$
\psi_{k}^{0} \rightarrow \psi^{0} \quad \text { as } \quad k \rightarrow \infty
$$

and for every $T>0$,

$$
\psi_{k} \rightarrow \psi \quad \text { uniformly on }[0, T] \quad \text { as } k \rightarrow \infty \text {, }
$$

where $\left(\psi, \psi^{0}\right)$ is a nontrivial pair of adjoint variables associated with $\left(u_{*}, x_{*}\right)$;

(iii) $\left(u_{*}, x_{*}\right)$ satisfies relations (1.4)-(1.6) of the core Pontryagin maximum principle in problem $(\mathrm{P})$ together with $\left(\psi, \psi^{0}\right)$;

(iv) the stationarity condition holds:

$$
H\left(x_{*}(t), t, \psi(t), \psi^{0}\right)=\psi^{0} \rho \int_{t}^{\infty} e^{-\rho s} g\left(x_{*}(s), u_{*}(s)\right) d s \quad \text { for all } \quad t \geq 0 .
$$

Proof. Lemma 1 and the Ascoli theorem (see, e.g., [19]) imply that, selecting a subsequence if needed, we get (3.11) and (3.12) for every $T>0$. By assumption, the sequence $\left\{\psi_{k}^{0}\right\}$ is bounded; therefore, selecting a subsequence if needed, we obtain (3.13) for some $\psi^{0} \geq 0$.

Now, our goal is to select a subsequence of $\left\{\left(u_{k}, x_{k}, \psi_{k}\right)\right\}$ such that for every $T>0,(3.14)$ holds and $\left(\psi, \psi^{0}\right)$ is a nontrivial pair of adjoint variables associated with $\left(u_{*}, x_{*}\right)$ (we do not change notation after the selection of a subsequence).

Consider the sequence $\left\{\psi_{k}\right\}$ restricted to $\left[0, T_{1}\right]$. Observing (3.6), taking into account the boundedness of the sequence $\left\{\psi_{k}(0)\right\}$ (see the assumptions of this lemma), using the Gronwall lemma (see, e.g., [25]), and selecting if needed a subsequence denoted further as $\left\{\psi_{k}^{1}\right\}$, we get that $\psi_{k}^{1} \rightarrow \psi^{1}$ uniformly on $\left[0, T_{1}\right]$ and $\dot{\psi}_{k}^{1} \rightarrow \dot{\psi}^{1}$ weakly in $L^{1}\left[0, T_{1}\right]$ as $k \rightarrow \infty$ for some absolutely continuous $\psi^{1}:\left[0, T_{1}\right] \rightarrow \mathbb{R}^{n}$; here and in what follows $L^{1}[0, T]=L^{1}\left([0, T], \mathbb{R}^{n}\right)(T>0)$.

Now consider the sequence $\left\{\psi_{k}^{1}\right\}$ restricted to $\left[0, T_{2}\right]$. Taking if necessary a subsequence $\left\{\psi_{k}^{2}\right\}$ of $\left\{\psi_{k}^{1}\right\}$, we get that $\psi_{k}^{2} \rightarrow \psi^{2}$ uniformly on $\left[0, T_{2}\right]$ and $\dot{\psi}_{k}^{2} \rightarrow \dot{\psi}^{2}$ weakly in $L^{1}\left[0, T_{2}\right]$ as $k \rightarrow \infty$ for some absolutely continuous $\psi^{2}:\left[0, T_{2}\right] \rightarrow \mathbb{R}^{n}$ whose restriction to $\left[0, T_{1}\right]$ coincides with $\psi^{1}$.

Repeating this procedure sequentially for $\left[0, T_{i}\right]$ with $i=3,4, \ldots$, we find that there exist absolutely continuous $\psi^{i}:\left[0, T_{i}\right] \rightarrow \mathbb{R}^{n}(i=1,2, \ldots)$ and $\psi_{k}^{i}:\left[0, T_{i}\right] \rightarrow \mathbb{R}^{n}$ $(i, k=1,2, \ldots)$ such that for every $i=1,2, \ldots$, the restriction of $\psi^{i+1}$ to $\left[0, T_{i}\right]$ is $\psi^{i}$, the restriction of the sequence $\left\{\psi_{k}^{i+1}\right\}$ to $\left[0, T_{i}\right]$ is a subsequence of $\left\{\psi_{k}^{i}\right\}$, and, moreover, $\psi_{k}^{i} \rightarrow \psi$ uniformly on $\left[0, T_{i}\right]$ and $\dot{\psi}_{k}^{i} \rightarrow \dot{\psi}^{i}$ weakly in $L^{1}\left[0, T_{i}\right]$ as $k \rightarrow \infty$. 
Define $\psi:[0, \infty) \mapsto \mathbb{R}^{n}$ so that the restriction of $\psi$ to $\left[0, T_{i}\right]$ is $\psi^{i}$ for every $i=1,2, \ldots$. Clearly, $\psi$ is absolutely continuous. Furthermore, without changing notation, for every $i=1,2, \ldots$ and every $k=1,2, \ldots$, we extend $\psi_{k}^{i}$ to $[0, \infty)$ so that the extended function is absolutely continuous and, moreover, the family $\dot{\psi}_{k}^{i}$ $(i, k=1,2, \ldots)$ is bounded in $L^{1}[0, T]$ for every $T>0$. Since $T_{i} \rightarrow \infty$ as $i \rightarrow \infty$, for every $T>0$, we get that $\psi_{k}^{k}$ converges to $\psi$ uniformly on $[0, T]$ and $\dot{\psi}_{k}^{k} \rightarrow \dot{\psi}$ weakly in $L^{1}[0, T]$ as $k \rightarrow \infty$. Simplifying notation, we again write $\psi_{k}$ instead of $\psi_{k}^{k}$ and note that for $\psi_{k},(3.6)$ holds $(k=1,2, \ldots)$. Thus, for every $T>0$, we have (3.14) and also get that $\dot{\psi}_{k} \rightarrow \dot{\psi}$ weakly in $L^{1}[0, T]$ as $k \rightarrow \infty$. These convergences together with equalities (3.6) and convergences (3.11) and (3.12) (holding for every $T>0$ ) yield that $\psi$ solves the adjoint equation (1.4). Thus, $\left(\psi, \psi^{0}\right)$ is a pair of adjoint variables associated with $\left(u_{*}, x_{*}\right)$ in problem $(\mathrm{P})$. The nontriviality of $\left(\psi, \psi^{0}\right)$ (see $\left.(1.5)\right)$ is ensured by (3.10).

For every $k=1,2, \ldots$, consider the maximum condition (3.7) and specify it as

$$
\begin{aligned}
& \left\langle f\left(x_{k}(t), u_{k}(t)\right), \psi_{k}(t)\right\rangle+\psi_{k}^{0} e^{-\rho t} g\left(x_{k}(t), u_{k}(t)\right)-\psi_{k}^{0} e^{-(\rho+1) t} \frac{\left\|u_{k}(t)-z_{k}(t)\right\|^{2}}{1+\sigma_{k}} \\
& \stackrel{\text { a.e. }}{=} \max _{u \in U}\left[\left\langle f\left(x_{k}(t), u\right), \psi_{k}(t)\right\rangle+\psi_{k}^{0} e^{-\rho t} g\left(x_{k}(t), u\right)-\psi_{k}^{0} e^{-(\rho+1) t} \frac{\left\|u-z_{k}(t)\right\|^{2}}{1+\sigma_{k}}\right] .
\end{aligned}
$$

Taking into account that $T_{k} \rightarrow \infty$ and $\sigma_{k} \rightarrow \infty$ as $k \rightarrow \infty$ and using convergences (3.13), (3.14), (3.11), and (3.12) (holding for every $T>0$ ), we obtain the maximum condition (1.6) as the limit of (3.7). Thus, $\left(u_{*}, x_{*}\right)$ satisfies the core Pontryagin maximum principle together with the pair $\left(\psi, \psi^{0}\right)$ of adjoint variables associated with $\left(u_{*}, x_{*}\right)$.

Now we specify (3.9) using the form of $\mathcal{H}_{k}$ (see (3.9)). We get

$$
\begin{aligned}
\frac{d}{d t} H_{k}\left(x_{k}(t), t, \psi_{k}(t), \psi_{k}^{0}\right) \stackrel{\text { a.e. }}{=} & \frac{\partial \mathcal{H}_{k}}{\partial t}\left(x_{k}(t), t, u_{k}(t), \psi_{k}(t), \psi_{k}^{0}\right) \\
\stackrel{\text { a.e. }}{=} & -\psi_{k}^{0} \rho e^{-\rho t}\left[g\left(x_{k}(t), u_{k}(t)\right)+(\rho+1) e^{-(\rho+1) t} \frac{\left\|u_{k}(t)-z_{k}(t)\right\|^{2}}{1+\sigma_{k}}\right] \\
& +2 \psi_{k}^{0} e^{-(\rho+1) t} \frac{\left\langle u_{k}(t)-z_{k}(t), \dot{z}_{k}(t)\right\rangle}{1+\sigma_{k}} .
\end{aligned}
$$

Take an arbitrary $t>0$ and an arbitrary $k$ such that $T_{k}>t$ and integrate the last equality over $\left[t, T_{k}\right]$ taking into account the boundary condition (3.5). We arrive at

$$
\begin{aligned}
H_{k}\left(x_{k}(t), t, \psi_{k}(t), \psi_{k}^{0}\right)= & \psi_{k}^{0} e^{-\rho T_{k}} \max _{u \in U}\left[g\left(x_{k}\left(T_{k}\right), u\right)-e^{-\rho T_{k}} \frac{\left\|u-z_{k}\left(T_{k}\right)\right\|^{2}}{1+\sigma_{k}}\right] \\
& -\psi_{k}^{0} \rho \int_{t}^{T_{k}} e^{-\rho s} g\left(x_{k}(s), u_{k}(s)\right) d s \\
& +\psi_{k}^{0}(\rho+1) \int_{t}^{T_{k}} e^{-(\rho+1) s} \frac{\left\|u_{k}(s)-z_{k}(s)\right\|^{2}}{1+\sigma_{k}} d s \\
& +2 \psi_{k}^{0} \int_{t}^{T_{k}} e^{-(\rho+1) s} \frac{\left\langle u_{k}(s)-z_{k}(s), \dot{z}_{k}(s)\right\rangle}{1+\sigma_{k}} d s .
\end{aligned}
$$


Now, we take the limit using convergences (3.13), (3.14), (3.11), and (3.12) (holding for every $T>0$ ) and also estimates (3.1)-(3.3). We end up with (3.15).

Corollary 1 below specifies Lemma 2 for the case where the Pontryagin maximum principle for problems $\left(\mathrm{P}_{k}\right)(k=1,2, \ldots)$ is taken in the normal form. We use the following formulation of the normal-form Pontryagin maximum principle for problems $\left(\mathrm{P}_{k}\right)(k=1,2, \ldots)$. Let an admissible pair $\left(u_{k}, x_{k}\right)$ be optimal in problem $\left(\mathrm{P}_{k}\right)$ for some $k$. Then there exists an adjoint variable $\psi_{k}$ associated with $\left(u_{k}, x_{k}\right)$ such that $\left(u_{k}, x_{k}\right)$ satisfies the normal-form core Pontryagin maximum principle (in problem $\left.\left(\mathrm{P}_{k}\right)\right)$ together with $\psi_{k}$, and the transversality condition (3.5) holds; here $\psi_{k}$ is a solution on $\left[0, T_{k}\right]$ of the normal-form adjoint equation associated with $\left(u_{k}, x_{k}\right)$ in problem $\left(\mathrm{P}_{k}\right)$, i.e.,

$$
\dot{\psi}_{k}(t) \stackrel{\text { a.e. }}{=}-\left[\frac{\partial f\left(x_{k}(t), u_{k}(t)\right)}{\partial x}\right]^{*} \psi_{k}(t)-e^{-\rho t} \frac{\partial g\left(x_{k}(t), u_{k}(t)\right)}{\partial x},
$$

and the fact that $\left(u_{k}, x_{k}\right)$ satisfies the normal-form core Pontryagin maximum principle, together with $\psi_{k}$, implies that the following maximum condition holds:

$$
\tilde{\mathcal{H}}_{k}\left(x_{k}(t), t, u_{k}(t), \psi(t)\right)=\tilde{H}_{k}\left(x_{k}(t), t, \psi_{k}(t)\right) \quad \text { for a.a. } t \in\left[0, T_{k}\right] ;
$$

here $\tilde{\mathcal{H}}_{k}$ and $\tilde{H}_{k}$, given by

$$
\begin{aligned}
\tilde{\mathcal{H}}_{k}(x, t, u, \psi) & =\langle f(x, u), \psi\rangle+e^{-\rho t} g(x, u)-e^{-(\rho+1) t} \frac{\left\|u-z_{k}(t)\right\|^{2}}{1+\sigma_{k}} \\
\tilde{H}_{k}(x, t, \psi) & =\sup _{u \in \tilde{U}} \tilde{\mathcal{H}}_{k}(x, t, \tilde{u}, \psi),
\end{aligned}
$$

are, respectively, the normal-form Hamilton-Pontryagin function and normal-form Hamiltonian in problem $\left(\mathrm{P}_{k}\right)$.

Corollary 1. Let assumptions (A1)-(A4) be satisfied; let $\left(u_{*}, x_{*}\right)$ be an admissible pair optimal in problem $(\mathrm{P})$; let $\left\{\left(P_{k}\right)\right\}(k=1,2, \ldots)$ be the sequence of problems associated with $u_{*}$; for every $k=1,2, \ldots$, let $\left(u_{k}, x_{k}\right)$ be an admissible pair optimal in problem $\left(\mathrm{P}_{k}\right)$; and for every $k=1,2, \ldots$, let $\psi_{k}$ be an adjoint variable associated with $\left(u_{k}, x_{k}\right)$ in problem $\left(\mathrm{P}_{k}\right)$ such that $\left(u_{k}, x_{k}\right)$ satisfies relations (3.16) and (3.17) of the normal-form core Pontryagin maximum principle in problem $\left(\mathrm{P}_{k}\right)$ together with $\psi_{k}$, and the transversality condition (3.5) holds. Finally, let the sequence $\left\{\psi_{k}(0)\right\}$ be bounded. Then there exists a subsequence of $\left\{\left(u_{k}, x_{k}, \psi_{k}\right)\right\}$, denoted again as $\left\{\left(u_{k}, x_{k}, \psi_{k}\right)\right\}$, such that

(i) for every $T>0,(3.11)$ and (3.12) hold;

(ii) for every $T>0,(3.14)$ holds where $\psi$ is an adjoint variable associated with $\left(u_{*}, x_{*}\right)$ in problem $(\mathrm{P})$;

(iii) $\left(u_{*}, x_{*}\right)$ satisfies relations (1.7) and (1.8) of the normal-form core Pontryagin maximum principle in problem $(\mathrm{P})$ together with $\psi$;

(iv) the normal-form stationarity condition holds:

$$
\tilde{H}\left(x_{*}(t), t, \psi(t)\right)=\rho \int_{t}^{\infty} e^{-\rho s} g\left(x_{*}(s), u_{*}(s)\right) d s \text { for all } t \geq 0 .
$$

Corollary 2. Let assumptions (A1)-(A4) be satisfied and let $\left(u_{*}, x_{*}\right)$ be an admissible pair optimal in problem $(\mathrm{P})$. Then there exists a pair $\left(\psi, \psi^{0}\right)$ of adjoint variables associated with $\left(u_{*}, x_{*}\right)$ such that

(i) $\left(u_{*}, x_{*}\right)$ satisfies relations (1.4)-(1.6) of the core Pontryagin maximum principle together with $\left(\psi, \psi^{0}\right)$, and

(ii) $\left(u_{*}, x_{*}\right)$ and $\left(\psi, \psi^{0}\right)$ satisfy the stationarity condition (3.15). 
Proof. Let $\left\{\left(\mathrm{P}_{k}\right)\right\}(k=1,2, \ldots)$ be the sequence of problems associated with $u_{*}$, and for every $k=1,2, \ldots$, let $\left(u_{k}, x_{k}\right)$ be an admissible pair optimal in problem $\left(\mathrm{P}_{k}\right)$. In accordance with the classical formulation of the Pontryagin maximum principle, for every $k=1,2, \ldots$, there exists a pair $\left(\psi_{k}, \psi_{k}^{0}\right)$ of adjoint variables associated with $\left(u_{k}, x_{k}\right)$ in problem $\left(\mathrm{P}_{k}\right)$ such that $\left(u_{k}, x_{k}\right)$ satisfies the core Pontryagin maximum principle together with $\left(\psi_{k}, \psi_{k}^{0}\right)$ and for every $k=1,2, \ldots, \psi_{k}^{0}>0$, and the transversality condition (3.5) holds.

Since $\psi_{k}^{0}>0$, the value $c_{k}=\left\|\psi_{k}(0)\right\|+\psi_{k}^{0}$ is positive. We keep the notation $\psi_{k}$ and $\psi_{k}^{0}$ for the normalized elements $\psi_{k} / c_{k}$ and $\psi_{k}^{0} / c_{k}$, thus achieving $\left\|\psi_{k}(0)\right\|+\psi_{k}^{0}=1$ and, clearly, preserving the transversality condition (3.5) and the fact that $\left(u_{k}, x_{k}\right)$ satisfies the core Pontryagin maximum principle (in problem $\left(\mathrm{P}_{k}\right)$ ) together with $\left(\psi_{k}, \psi_{k}^{0}\right)(k=1,2, \ldots)$. Now, the sequences $\left\{\psi_{k}(0)\right\}$ and $\left\{\psi_{k}^{0}\right\}$ are bounded and (3.10) holds with $a=1$. Thus, the sequence $\left\{\left(u_{k}, x_{k}, \psi_{k}, \psi_{k}^{0}\right)\right\}$ satisfies all the assumptions of Lemma 2. By Lemma 2 there exists a subsequence of $\left\{\left(u_{k}, x_{k}, \psi_{k}, \psi_{k}^{0}\right)\right\}$, denoted again as $\left\{\left(u_{k}, x_{k}, \psi_{k}, \psi_{k}^{0}\right)\right\}$, such that for the pairs $\left(\psi_{k}, \psi_{k}^{0}\right)$ of adjoint variables, convergences (3.13) and (3.14) hold with an arbitrary $T>0$; the limit element $\left(\psi, \psi^{0}\right)$ is a nontrivial pair of adjoint variables associated with $\left(u_{*}, x_{*}\right)$ in problem $(\mathrm{P}) ;\left(u_{*}, x_{*}\right)$ satisfies the core Pontryagin maximum principle in problem $(\mathrm{P})$ together with $\left(\psi, \psi^{0}\right)$; and, finally, $\left(u_{*}, x_{*}\right)$ and $\left(\psi, \psi^{0}\right)$ satisfy the stationarity condition (3.15).

It is easy to see that in the framework of problem $(\mathrm{P})$ the necessary optimality conditions given by Corollary 2 are equivalent to those stated in [31]. Indeed, relation (3.15) implies the asymptotic stationarity condition introduced in [31]:

$$
\lim _{t \rightarrow \infty} H\left(x_{*}(t), t, \psi(t), \psi^{0}\right)=0 ;
$$

on the other hand, if in problem $(\mathrm{P}),\left(u_{*}, x_{*}\right)$ satisfies the core Pontryagin maximum principle together with $\left(\psi, \psi^{0}\right)$, then (3.19) implies (3.15). One can, however, anticipate that beyond setting $(\mathrm{P})$ (for example, for problems with nonsmooth data), condition (3.15) complementing the core Pontryagin maximum principle can be substantially stronger than (3.19).

Note that in the problem considered in Example 1 the usage of the core Pontryagin maximum principle (Theorem 1) does not lead to the specification of an optimal control, whereas it can be shown that the latter control is determined uniquely if one applies the core Pontryagin maximum principle together with (3.15) (Corollary 2).

As an example given in [31] shows, under the assumptions of Corollary 2, the nontriviality condition (1.5) can hold with $\psi^{0}=0$; i.e., problem $(\mathrm{P})$ can be abnormal. Below we find additional assumptions excluding abnormality of problem $(\mathrm{P})$.

4. Normal-form maximum principle with positive adjoint variables. In this section, we suggest an assumption that excludes abnormality of problem (P), i.e., ensures that for problem $(\mathrm{P})$, the normal-form Pontryagin maximum principle (see section 1) provides a necessary condition of optimality. Moreover, the basic result of this section formulated in Theorem 2 states that all the coordinates of the adjoint variable $\psi$ in the Pontryagin maximum principle are necessarily positive-valued. Based on Theorem 2, we formulate conditions ensuring that the core Pontryagin maximum principle is complemented by the transversality conditions discussed in section 2 . The proof of Theorem 2 is based on Corollary 1.

In what follows, the notation $a>0$ (respectively, $a \geq 0$ ) for a vector $a \in \mathbb{R}^{n}$ designates that all coordinates of $a$ are positive (respectively, nonnegative). Similarly, the notation $A>0$ (respectively, $A \geq 0$ ) for a matrix $A$ designates that all elements of $A$ are positive (respectively, nonnegative). 
The assumption complementing (A1)-(A4) is the following.

(A5) For every admissible pair $(u, x)$ and for a.a. $t \geq 0$, one has

$$
\frac{\partial g(x(t), u(t))}{\partial x}>0 \text { and } \frac{\partial f^{i}(x(t), u(t))}{\partial x^{j}} \geq 0 \quad \text { for all } \quad i, j: i \neq j .
$$

In typical models of regulated economic growth the coordinates of the state vector $x$ represent positive-valued production factors. Normally it is assumed that the utility flow and the rate of growth of every production factor increase as all the production factors grow. In terms of problem $(\mathrm{P})$, this implies that the integrand $g(x, u)$ in the goal functional (1.3), together with every coordinate of the right-hand side $f(x, u)$ of the system equation (1.1), is monotonically increasing in every coordinate of $x$. These monotonicity properties (specified so that $g(x, u)$ is strictly increasing in every coordinate of $x$ ) imply that assumption (A5) is satisfied. Note that the utility flow and the rates of growth of the production factors are normally positive, implying $g(x, u)>0$ and $f(x, u)>0$. The latter assumptions as well as the assumption $x>0$ mentioned earlier appear in different combinations in the formulations of the results of this section.

The next theorem strengthens Theorem 1 under assumption (A5) and some positivity assumptions for $f$.

THEOREM 2. Let assumptions (A1)-(A5) be satisfied. There exists a $u_{0} \in U$ such that $f\left(x_{0}, u_{0}\right)>0$, and for every admissible pair $(u, x)$, it holds that $f(x(t), u(t)) \geq 0$ for a.a. $t \geq 0$. Let $\left(u_{*}, x_{*}\right)$ be an admissible pair optimal in problem $(\mathrm{P})$. Then there exists an adjoint variable $\psi$ associated with $\left(u_{*}, x_{*}\right)$ such that

(i) $\left(u_{*}, x_{*}\right)$ satisfies relations (1.7) and (1.8) of the normal-form core Pontryagin maximum principle together with $\psi$;

(ii) $\left(u_{*}, x_{*}\right)$ and $\psi$ satisfy the normal-form stationarity condition (3.18);

(iii)

$$
\psi(t)>0 \text { for all } t \geq 0 \text {. }
$$

Proof. Let $\left\{\left(\mathrm{P}_{k}\right)\right\}(k=1,2, \ldots)$ be the sequence of problems associated with $u_{*}$ and for every $k=1,2, \ldots$, let $\left(u_{k}, x_{k}\right)$ be an admissible pair optimal in problem $\left(\mathrm{P}_{k}\right)$. In accordance with the classical formulation of the normal-form Pontryagin maximum principle, for every $k=1,2, \ldots$, there exists an adjoint variable $\psi_{k}$ associated with $\left(u_{k}, x_{k}\right)$ in problem $\left(\mathrm{P}_{k}\right)$ such that $\left(u_{k}, x_{k}\right)$ satisfies the normal-form core Pontryagin maximum principle (in problem $\left(\mathrm{P}_{k}\right)$ ) together with $\psi_{k}$ and for every $k=1,2, \ldots$, the transversality condition (3.5) holds.

Observing assumption (A5), the adjoint equation resolved by $\psi_{k}$ (see (3.16)), and transversality condition (3.5) for $\psi_{k}$, we easily find that $\psi_{k}(t)>0$ for all $t$ sufficiently close to $T_{k}$. Let us show that

$$
\psi_{k}(t)>0 \text { for all } t \in\left[0, T_{k}\right) .
$$

Suppose the contrary. Then, for some $k$, there exists a $\tau \in\left[0, T_{k}\right)$ such that at least one coordinate of the vector $\psi_{k}(\tau)$ vanishes. Let $\xi$ be the maximum of all such $\tau \in\left[0, T_{k}\right)$, and let $i \in\{1,2, \ldots, n\}$ be such that $\psi_{k}^{i}(\xi)=0$. Then,

$$
\psi_{k}(t)>0 \text { for all } t \in\left(\xi, T_{k}\right)
$$

and for all $t \in\left[\xi, T_{k}\right]$, we have

$$
\psi_{k}^{i}(t)=-\int_{\xi}^{t}\left\langle\frac{\partial f^{i}\left(x_{k}(s), u_{k}(s)\right)}{\partial x}, \psi_{k}(s)\right\rangle d s-\int_{\xi}^{t} e^{-\rho s} \frac{\partial g^{i}\left(x_{k}(s), u_{k}(s)\right)}{\partial x} d s .
$$


The latter equation and assumption (A5) imply that there is an $\epsilon>0$ such that $\psi_{k}^{i}(t)<0$ for all $t \in(\xi, \xi+\epsilon)$, which contradicts (4.3). The contradiction proves (4.2).

Let us show that the sequence $\left\{\psi_{k}(0)\right\}$ is bounded. The equation for $\psi_{k}$ (see (3.16)) and maximum condition (3.17) yield

$$
\begin{aligned}
\frac{d}{d t} \tilde{H}_{k}\left(x_{k}(t), t, \psi_{k}(t)\right) \stackrel{\text { a.e. }}{=} & \frac{\partial \tilde{\mathcal{H}}_{k}}{\partial t}\left(x_{k}(t), t, \tilde{u}_{k}(t), \psi_{k}(t)\right) \\
\stackrel{\text { a.e. }}{=} & -\rho e^{-\rho t} g\left(x_{k}(t), u_{k}(t)\right)+(\rho+1) e^{-(\rho+1) t} \frac{\left\|u_{k}(t)-z_{k}(t)\right\|^{2}}{1+\sigma_{k}} \\
& +2 e^{-(\rho+1) t} \frac{\left\langle u_{k}(t)-z_{k}(t), \dot{z}_{k}(t)\right\rangle}{1+\sigma_{k}} .
\end{aligned}
$$

Integrating over $\left[0, T_{k}\right]$ and using the transversality condition (3.5), we arrive at

$$
\begin{aligned}
\tilde{H}_{k}\left(x_{0}, 0, \psi_{k}(0)\right)= & e^{-\rho T_{k}} \max _{u \in U}\left[g\left(x_{k}\left(T_{k}\right), u\right)-e^{-T_{k}} \frac{\left\|u-z_{k}\left(T_{k}\right)\right\|^{2}}{1+\sigma_{k}}\right] \\
& +\rho \int_{0}^{T_{k}} e^{-\rho t} g\left(x_{k}(t), u_{k}(t)\right) d t \\
& -(\rho+1) \int_{0}^{T_{k}} e^{-(\rho+1) t} \frac{\left\|u_{k}(t)-z_{k}(t)\right\|^{2}}{1+\sigma_{k}} d t \\
& -2 \int_{0}^{T_{k}} e^{-(\rho+1) t} \frac{\left\langle u_{k}(t)-z_{k}(t), \dot{z}_{k}(t)\right\rangle}{1+\sigma_{k}} d t .
\end{aligned}
$$

This, together with (3.1)-(3.3), implies that $\tilde{H}_{k}\left(x_{0}, 0, \psi_{k}(0)\right) \leq M$ for some $M>0$ and all $k=1,2, \ldots$ Hence, by virtue of

$$
\left\langle f\left(x_{0}, u_{0}\right), \psi_{k}(0)\right\rangle+g\left(x_{0}, u_{0}\right)-\frac{\left\|u_{0}-z_{k}(0)\right\|^{2}}{1+\sigma_{k}} \leq \tilde{H}_{k}\left(x_{0}, 0, \psi_{k}(0)\right),
$$

we have

$$
\left\langle f\left(x_{0}, u_{0}\right), \psi_{k}(0)\right\rangle \leq M+\left|g\left(x_{0}, u_{0}\right)\right|+(2|U|+1)^{2},
$$

where $|U|=\max _{u \in U}\|u\|$. The latter estimate, assumption $f\left(x_{0}, u_{0}\right)>0$, and (4.2) yield that the sequence $\left\{\psi_{k}(0)\right\}$ is bounded.

Therefore, the sequence $\left\{\left(u_{k}, x_{k}, \psi_{k}\right)\right\}$ satisfies all the assumptions of Corollary 1. By Corollary 1, there exists a subsequence of $\left\{\left(u_{k}, x_{k}, \psi_{k}\right)\right\}$, denoted again as $\left\{\left(u_{k}, x_{k}, \psi_{k}\right)\right\}$, such that for every $T>0$, one has convergence (3.14) for the adjoint variables $\psi_{k}$, where the limit element $\psi$ is an adjoint variable associated with $\left(u_{*}, x_{*}\right)$ in problem $(\mathrm{P}) ;\left(u_{*}, x_{*}\right)$ satisfies the normal-form core Pontryagin maximum principle in problem $(\mathrm{P})$ together with $\psi$; and, finally, $\left(u_{*}, x_{*}\right)$ and $\psi$ satisfy the normal-form asymptotic stationarity condition (3.18). Thus, for $\left(u_{*}, x_{*}\right)$ and $\psi$, statements (i) and (ii) are proved.

From (3.14) and (4.2) it follows that $\psi(t) \geq 0$ for all $t \geq 0$. Assumption (A5) and the fact that $\psi$ solves the adjoint equation (1.7) imply (4.1), thus proving (iii).

Now, we formulate conditions coupling the normal-form core Pontryagin maximum principle and the transversality conditions discussed in section 2 . 
Corollary 3. Let the assumptions of Theorem 2 be satisfied and

$$
f\left(x_{*}(t), u_{*}(t)\right) \geq a_{1} \quad \text { for a.a. } \quad t \geq 0,
$$

where $a_{1}>0$. Then there exists an adjoint variable $\psi$ associated with $\left(u_{*}, x_{*}\right)$ such that statements (i), (ii), and (iii) of Theorem 2 hold true and, moreover, $\psi$ satisfies the transversality condition

$$
\lim _{t \rightarrow \infty} \psi(t)=0 .
$$

Proof. By Theorem 2, there exists an adjoint variable $\psi$ associated with $\left(u_{*}, x_{*}\right)$ such that statements (i), (ii), and (iii) of Theorem 2 hold true. Let us prove (4.6). From (3.15) and (4.5) we get

$$
\lim _{t \rightarrow \infty}\left\langle a_{1}, \psi(t)\right\rangle \leq \lim _{t \rightarrow \infty} \max _{u \in U}\left\langle f\left(x_{*}(t), u\right), \psi(t)\right\rangle=0 ;
$$

the latter, together with (4.1), implies (4.6).

COROllary 4. Let the assumptions of Theorem 2 be satisfied, and let

$$
\begin{gathered}
x_{0} \geq 0 \\
g\left(x_{*}(t), u_{*}(t)\right) \geq 0 \quad \text { for a.a. } t \geq 0 ;
\end{gathered}
$$

and

$$
\frac{\partial f\left(x_{*}(t), u_{*}(t)\right)}{\partial x} \geq A \quad \text { for a.a. } \quad t \geq 0,
$$

where $A$ is a matrix of order $n$ such that $A>0$. Then there exists an adjoint variable $\psi$ associated with $\left(u_{*}, x_{*}\right)$ such that statements (i), (ii), and (iii) of Theorem 2 hold true and, moreover, $\psi$ satisfies the transversality condition

$$
\lim _{t \rightarrow \infty}\left\langle x_{*}(t), \psi(t)\right\rangle=0
$$

Proof. By Theorem 2, there exists an adjoint variable $\psi$ associated with $\left(u_{*}, x_{*}\right)$ such that statements (i), (ii), and (iii) of Theorem 2 hold true. Let us prove (4.10). The system equation (1.1) and normal-form adjoint equation (1.7) yield

$$
\begin{aligned}
\frac{d}{d t}\left\langle x_{*}(t), \psi(t)\right\rangle= & \left\langle f\left(x_{*}(t), u_{*}(t)\right), \psi(t)\right\rangle \\
& -\left\langle x_{*}(t),\left[\frac{\partial f\left(x_{*}(t), u_{*}(t)\right)}{\partial x}\right]^{*} \psi(t)\right\rangle \\
& -e^{-\rho t}\left\langle x_{*}(t), \frac{\partial g\left(x_{*}(t), u_{*}(t)\right)}{\partial x}\right\rangle \text { for a.a. } t \geq 0
\end{aligned}
$$

From (4.7), assumption (A5), and (4.8), it follows that

$$
-e^{-\rho t}\left\langle x_{*}(t), \frac{\partial g\left(x_{*}(t), u_{*}(t)\right)}{\partial x}\right\rangle \leq 0 \leq e^{-\rho t} g\left(x_{*}(t), u_{*}(t)\right) .
$$


Taking this into account and using assumption (A5), the normal-form maximum condition (1.8), and assumption (4.9), we continue (4.11) as follows:

$$
\begin{aligned}
\frac{d}{d t}\left\langle x_{*}(t), \psi(t)\right\rangle \leq & \left\langle f\left(x_{*}(t), u_{*}(t)\right), \psi(t)\right\rangle \\
& -\left\langle x_{*}(t),\left[\frac{\partial f\left(x_{*}(t), u_{*}(t)\right)}{\partial x}\right]^{*} \psi(t)\right\rangle+e^{-\rho t} g\left(x_{*}(t), u_{*}(t)\right) \\
\leq & -\left\langle A x_{*}(t), \psi(t)\right\rangle+\tilde{H}\left(x_{*}(t), t, \psi(t)\right) \quad \text { for a.a. } \quad t \geq 0 .
\end{aligned}
$$

Therefore, by (4.9), for some $\theta>0$, we have

$$
\frac{d}{d t}\left\langle x_{*}(t), \psi(t)\right\rangle \leq-\theta\left\langle x_{*}(t), \psi(t)\right\rangle+\alpha(t),
$$

where

$$
\alpha(t)=\tilde{H}\left(x_{*}(t), t, \psi(t)\right) \rightarrow 0 \quad \text { as } \quad t \rightarrow \infty
$$

(see (3.18)). Then, taking into account (4.7) and (4.1), we get

$$
0 \leq\left\langle x_{*}(t), \psi(t)\right\rangle \leq e^{-\theta t}\left\langle x_{0}, \psi(0)\right\rangle+e^{-\theta t} \int_{0}^{t} e^{\theta s} \alpha(s) d s .
$$

Furthermore,

$$
\begin{aligned}
\dot{\alpha}(t) & =\frac{d}{d t} \tilde{H}\left(x_{*}(t), t, \psi(t)\right)=\frac{\partial}{\partial t} \tilde{\mathcal{H}}\left(x_{*}(t), t, u_{*}(t), \psi(t)\right) \\
& =-\rho e^{-\rho t} g\left(x_{*}(t), u_{*}(t)\right) \leq 0 \quad \text { for a.a. } \quad t \geq 0
\end{aligned}
$$

(here we used (4.8)). Therefore,

$$
\int_{0}^{t} e^{\theta s} \alpha(s) d s=\frac{1}{\theta}\left[e^{\theta t} \alpha(t)-\alpha(0)\right]+\frac{1}{\theta} \int_{0}^{t} e^{\theta s} \dot{\alpha}(s) d s \leq \frac{1}{\theta}\left(e^{\theta t} \alpha(t)-\alpha(0)\right) .
$$

Substituting this estimate into (4.12), we get

$$
0 \leq\left\langle x_{*}(t), \psi(t)\right\rangle \leq e^{-\theta t}\left\langle x_{0}, \psi(0)\right\rangle+e^{-\theta t} \frac{1}{\theta}\left[e^{\theta t} \alpha(t)-\alpha(0)\right] \rightarrow 0 \quad \text { as } \quad t \rightarrow \infty .
$$

The next theorem is, to a certain extent, an inversion of Theorem 2. It adjoins works treating the Pontryagin maximum principle as a key component in sufficient conditions of optimality. Within the finite-horizon setting, this line of analysis was initiated in [30]. In [1] the approach was extended to infinite-horizon optimal control problems.

THEOREM 3. Let assumptions (A1)-(A5) be satisfied, $x_{0} \geq 0$, and for every admissible pair $(u, x)$, it holds that $f(x(t), u(t)) \geq 0$ and $g(x(t), u(t)) \geq 0$ for a.a. $t \geq 0$. Let $\left(u_{*}, x_{*}\right)$ be an admissible pair satisfying (4.9) with some $A>0$, and there exists an adjoint variable $\psi$ associated with $\left(u_{*}, x_{*}\right)$ such that statements (i), (ii), and (iii) of Theorem 2 hold true. Finally, let the set $G$ be convex and the function $x \mapsto \tilde{H}(x, t, \psi(t)): G \mapsto \mathbb{R}^{1}$ be concave for every $t \geq 0$. Then, the admissible pair $\left(u_{*}, x_{*}\right)$ is optimal in problem $(\mathrm{P})$. 
We omit the proof, which is similar to the proofs given in [2] and [36].

Combining Corollary 4 and Theorem 3, we arrive at the following optimality criterion for problem $(\mathrm{P})$.

CoROllary 5. Let assumptions (A1)-(A5) be satisfied, $x_{0} \geq 0$, and there exists $a u_{0} \in U$ such that $f\left(x_{0}, u_{0}\right)>0$. For every admissible pair $(u, x)$ it holds that $f(x(t), u(t)) \geq 0, g(x(t), u(t)) \geq 0$, and $\partial f(x(t), u(t)) / \partial x \geq A$ for a.a. $t \geq 0$ with some $A>0$. Let, finally, the set $G$ be convex and the function $x \mapsto \tilde{H}(x, t, \psi): G \mapsto \mathbb{R}^{1}$ be concave for every $t \geq 0$ and for every $\psi>0$. Then, an admissible pair $\left(u_{*}, x_{*}\right)$ is optimal in problem $(\mathrm{P})$ if and only if there exists an adjoint variable $\psi$ associated with $\left(u_{*}, x_{*}\right)$ such that statements (i), (ii), and (iii) of Theorem 2 hold true and the transversality condition (4.10) is satisfied.

5. Case of dominating discount. In [12], infinite-horizon necessary optimality conditions involving the normal-form core Pontryagin maximum principle and an integral characterization of global behavior of the adjoint variable were stated for problems with sufficiently large (dominating) discount factors; in this work the control system was assumed to be linear. In this section, we consider problem (P) for the nonlinear control system (1.1) and apply the approximation scheme developed in section 3 in the case of the dominating discount. If system (1.1) is linear, the basic statement of this section (Theorem 4) leads to a formulation of the Pontryagin maximum principle (Corollary 7), which is stronger than that given in [12].

Following [12], we posit the next growth constraint on $g$.

(A6) There exist a $\kappa \geq 0$ and an $r \geq 0$ such that

$$
\left\|\frac{\partial g(x, u)}{\partial x}\right\| \leq \kappa\left(1+\|x\|^{r}\right) \text { for all } x \in G \text { and for all } u \in U .
$$

Given an admissible pair $(u, x)$, we denote by $Y_{(u, x)}$ the normalized fundamental matrix for the linear differential equation

$$
\dot{y}(t)=\frac{\partial f(x(t), u(t))}{\partial x} y(t) ;
$$

more specifically, $Y_{(x, u)}$ is the $n \times n$ matrix-valued function on $[0, \infty)$ whose columns $y_{i}(i=1, \ldots, n)$ are the solutions to $(5.1)$ such that $y_{i}^{j}(0)=\delta_{i, j}(i, j=1, \ldots, n)$, where $\delta_{i, i}=1$ and $\delta_{i, j}=0$ for $i \neq j$; for every $t \geq 0,\left\|Y_{(u, x)}(t)\right\|$ stands for the standard norm of $Y_{(u, x)}(t)$ as a linear operator in $\mathbb{R}^{n}$. Similarly, given an admissible pair $(u, x)$, we denote by $Z_{(u, x)}$ the normalized fundamental matrix for the linear differential equation

$$
\dot{z}(t)=-\left[\frac{\partial f(x(t), u(t))}{\partial x}\right]^{*} z(t) .
$$

Note that

$$
\left[Z_{(u, x)}(t)\right]^{-1}=\left[Y_{(u, x)}(t)\right]^{*} .
$$

We introduce the following growth assumption.

(A7) There exist a $\lambda \in \mathbb{R}^{1}$, a $C_{1} \geq 0$, a $C_{2} \geq 0$, and a $C_{3} \geq 0$ such that for every admissible pair $(u, x)$, one has

$$
\|x(t)\| \leq C_{1}+C_{2} e^{\lambda t} \quad \text { for all } \quad t \geq 0
$$

and

$$
\left\|Y_{(u, x)}(t)\right\| \leq C_{3} e^{\lambda t} \quad \text { for all } \quad t \geq 0
$$


It is easily seen that assumption (A6) implies that there exist a $C_{4} \geq 0$ and a $C_{5} \geq 0$ such that for every admissible pair $(u, x)$, one has

$$
|g(x(t), u(t))| \leq C_{4}+C_{5}\|x(t)\|^{r+1} \quad \text { for all } \quad t \geq 0 .
$$

Furthermore, (A7) and (5.3) imply that

$$
e^{-\rho t}|g(x(t), u(t))| \leq C_{6} e^{-\rho t}+C_{7} e^{-(\rho-(r+1) \lambda) t}
$$

holds for every admissible pair $(u, x)$ with $C_{6} \geq 0$ and $C_{7} \geq 0$ not depending on $(u, x)$. Therefore, if $\rho>0$, then assumptions (A6) and (A7) imply (A4), provided $\rho>(r+1) \lambda$. The latter inequality implies that the discount parameter $\rho$ in the goal functional (1.3) dominates the growth parameters $r$ and $\lambda$ (see (A6) and (A7)), which is a counterpart of a condition assumed in [12].

The proof of the next result is based on Corollary 1.

THEOREM 4. Let assumptions (A1)-(A4), (A6), and (A7) be satisfied and let $\rho>(r+1) \lambda$. Let $\left(u_{*}, x_{*}\right)$ be an admissible pair optimal in problem $(\mathrm{P})$. Then there exists an adjoint variable $\psi$ associated with $\left(u_{*}, x_{*}\right)$ such that

(i) $\left(u_{*}, x_{*}\right)$ satisfies relations (1.7) and (1.8) of the normal-form core Pontryagin maximum principle together with $\psi$;

(ii) $\left(u_{*}, x_{*}\right)$ and $\psi$ satisfy the normal-form stationarity condition (3.18);

(iii) for every $t \geq 0$, the integral

$$
I_{*}(t)=\int_{t}^{\infty} e^{-\rho s}\left[Z_{*}(s)\right]^{-1} \frac{\partial g\left(x_{*}(s), u_{*}(s)\right)}{\partial x} d s,
$$

where $Z_{*}=Z_{\left(u_{*}, x_{*}\right)}$, converges absolutely and

$$
\psi(t)=Z_{*}(t) I_{*}(t) .
$$

Proof. Let $\left\{\left(\mathrm{P}_{k}\right)\right\}(k=1,2, \ldots)$ be the sequence of problems associated with $u_{*}$ and for every $k=1,2, \ldots$, let $\left(u_{k}, x_{k}\right)$ be an admissible pair optimal in problem $\left(\mathrm{P}_{k}\right)$. In accordance with the classical formulation of the normal-form Pontryagin maximum principle, for every $k=1,2, \ldots$, there exists an adjoint variable $\psi_{k}$ associated with $\left(u_{k}, x_{k}\right)$ in problem $\left(\mathrm{P}_{k}\right)$ such that $\left(u_{k}, x_{k}\right)$ satisfies the normal-form core Pontryagin maximum principle (in problem $\left(\mathrm{P}_{k}\right)$ ) together with $\psi_{k}$ and for every $k=1,2, \ldots$, the transversality condition (3.5) holds.

Let us show that the sequence $\left\{\psi_{k}(0)\right\}$ is bounded. Using the standard representation of the solution $\psi_{k}$ to the linear normal-form adjoint equation (3.16) with the zero-boundary condition (3.5) through the fundamental matrix $Z_{k}=Z_{\left(u_{k}, x_{k}\right)}$ of the corresponding linear homogeneous equation (see, e.g., [25]), we get

$$
\psi_{k}(0)=\int_{0}^{T_{k}} e^{-\rho s}\left[Z_{k}(s)\right]^{-1} \frac{\partial g\left(x_{k}(s), u_{k}(s)\right)}{\partial x} d s .
$$

Therefore, due to (5.2),

$$
\left\|\psi_{k}(0)\right\| \leq \int_{0}^{T_{k}} e^{-\rho s}\left\|Y_{\left(x_{k}, u_{k}\right)}(s)\right\|\left\|\frac{\partial g\left(x_{k}(s), u_{k}(s)\right)}{\partial x}\right\| d s,
$$

and due to assumptions (A6) and (A7),

$$
\left\|\psi_{k}(0)\right\| \leq \int_{0}^{T_{k}}\left(C_{8} e^{-(\rho-\lambda) s}+C_{9} e^{-(\rho-(r+1) \lambda) s}\right) d s,
$$


where $C_{8} \geq 0$ and $C_{9} \geq 0$ do not depend on $k$. Now the assumption $\rho>(r+1) \lambda$ implies that the sequence $\left\{\psi_{k}(0)\right\}$ is bounded.

Therefore, the sequence $\left\{\left(u_{k}, x_{k}, \psi_{k}\right)\right\}$ satisfies all the assumptions of Corollary 1. By Corollary 1 , there exists a subsequence of $\left\{\left(u_{k}, x_{k}, \psi_{k}\right)\right\}$, denoted again as $\left\{\left(u_{k}, x_{k}, \psi_{k}\right)\right\}$, such that for every $T>0$, one has convergences (3.11) and (3.12) for the admissible pairs $\left(u_{k}, x_{k}\right)$ and convergence (3.14) for the adjoint variables $\psi_{k}$, where the limit element $\psi$ is an adjoint variable associated with $\left(u_{*}, x_{*}\right)$ in problem $(\mathrm{P}) ;\left(u_{*}, x_{*}\right)$ satisfies the normal-form core Pontryagin maximum principle in problem (P) together with $\psi$; and, finally, $\left(u_{*}, x_{*}\right)$ and $\psi$ satisfy the normal-form stationarity condition (3.18). Thus, for $\left(u_{*}, x_{*}\right)$ and $\psi$, statements (i) and (ii) are proved.

Consider the integral $I_{*}(t)$ in (5.4) for an arbitrary $t \geq 0$. Convergences (3.11) and (3.12) imply

$$
Z_{k}(s) \rightarrow Z_{*}(s) \text { for all } s \geq 0 .
$$

Hence,

$$
\begin{aligned}
I_{*}(t) & =\lim _{T \rightarrow \infty} \int_{t}^{T} e^{-\rho s}\left[Z_{*}(s)\right]^{-1} \frac{\partial g\left(x_{*}(s), u_{*}(s)\right)}{\partial x} d s \\
& =\lim _{T \rightarrow \infty} \lim _{k \rightarrow \infty} \int_{t}^{T} e^{-\rho s}\left[Z_{k}(s)\right]^{-1} \frac{\partial g\left(x_{k}(s), u_{k}(s)\right)}{\partial x} d s .
\end{aligned}
$$

Furthermore, from (5.2) and (A7) it follows that for all $s \geq 0$,

$$
e^{-\rho t}\left\|\left[Z_{k}(s)\right]^{-1}\right\|\left\|\frac{\partial g\left(x_{k}(s), u_{k}(s)\right)}{\partial x}\right\| \leq C_{10} e^{-(\rho-\lambda) s}+C_{11} e^{-(\rho-(r+1) \lambda) s}
$$

with some positive $C_{10}$ and $C_{11}$. Therefore, $I_{*}(t)$ converges absolutely. Let us prove (5.5). Integrate the adjoint equation for $\psi_{k}$ (see (3.16)) over $\left[t, T_{k}\right]$ assuming that $k$ is large enough (i.e., $T_{k} \geq t$ ) and taking into account the transversality condition (3.5). We get

$$
\psi_{k}(t)=Z_{k}(t) \int_{t}^{T_{k}} e^{-\rho s} Z_{k}^{-1}(s) \frac{\partial g\left(x_{k}(s), u_{k}(s)\right)}{\partial x} d s .
$$

Convergences (3.11) and (3.12) (holding for every $T>0$ ) imply that $x_{k}(s) \rightarrow x_{*}(s)$ for all $s \geq 0$ and $u_{k}(s) \rightarrow u_{*}(s)$ for a.a. $s \geq 0$. The latter convergences, convergences (5.6) and (3.14), and the absolute convergence of the integral $I_{*}(t)$ yield that the desired equality (5.5) is the limit of (5.7) with $k \rightarrow \infty$. Statement (iii) is proved.

Now we recall some facts from the stability theory (see [18], [20]).

Consider a linear differential equation

$$
\dot{x}(t)=A(t) x(t) .
$$

Here $t \in[0, \infty), x \in \mathbb{R}^{n}$, and the components of the real $n \times n$ matrix function $A$ are measurable and bounded.

Let $x$ be a nonzero solution to (5.8). Then, a number

$$
\lambda=\limsup _{t \rightarrow \infty} \frac{1}{t} \ln \|x(t)\|
$$


is said to be the Lyapunov characteristic number of $x$. Note that the characteristic number $\lambda$ was defined by A. M. Lyapunov with the opposite sign [29].

The Lyapunov characteristic number of any nonzero solution to (5.8) is finite. The set of the characteristic numbers for all nonzero solutions to (5.8) is called the Lyapunov spectrum of (5.8). The Lyapunov spectrum of (5.8) has no more than $n$ elements. A fundamental system $x_{1}, \ldots, x_{n}$ of solutions to (5.8) is said to be normal if the sum of their characteristic numbers is minimal in the set of all fundamental systems of solutions to (5.8). A normal fundamental system always exists. If $x_{1}, \ldots, x_{n}$ is a normal fundamental system of solutions to (5.8), then the characteristic numbers for $x_{1}, \ldots, x_{n}$ cover the Lyapunov spectrum of (5.8) (for different $x_{j}$ and $x_{k}$ the Lyapunov characteristic numbers may coincide). Any normal fundamental system contains the same number $n_{s}$ of solutions to (5.8) with characteristic number $\lambda_{s}, 1 \leq s \leq l, l \leq n$, from the Lyapunov spectrum of (5.8).

Let $\sigma=\sum_{s=1}^{l} n_{s} \lambda_{s}$ be the sum of all numbers $\lambda_{1}, \ldots, \lambda_{l}$ from the Lyapunov spectrum of (5.8) taken according to their multiplicity. Equation (5.8) is called regular if

$$
\sigma=\liminf _{t \rightarrow \infty} \frac{1}{t} \int_{0}^{t} \operatorname{tr} A(s) d s,
$$

where $\operatorname{tr} A(s)$ is the trace of matrix $A(s)$. If (5.8) is regular, then for every $\epsilon>0$, the Cauchy matrix $(s, t) \mapsto K(s, t)$ for $(5.8)$ satisfies

$$
\|K(s, t)\| \leq \kappa_{1} e^{\bar{\lambda}(s-t)+\epsilon s} \quad \text { for all } t \geq 0 \text { and all } s \geq t,
$$

where $\bar{\lambda}$ is the maximum element in the Lyapunov spectrum of (5.8) and $\kappa_{1} \geq 0$ is a constant depending only on $\epsilon$ (see [20]).

Corollary 6 . Let the assumptions of Theorem 4 be satisfied, let the linear differential equation

$$
\dot{y}(t)=\frac{\partial f\left(x_{*}(t), u_{*}(t)\right)}{\partial x} y(t)
$$

be regular, and let $\lambda \geq \bar{\lambda}$, where $\bar{\lambda}$ is the maximum element in the Lyapunov spectrum of (5.10). Then for every $\epsilon>0$, it holds that

$$
\|\psi(t)\| \leq \kappa_{2}\left(e^{-\rho t+\epsilon t}+e^{-(\rho-r \lambda) t+\epsilon t}\right) \quad \text { for all } t \geq 0,
$$

where $\kappa_{2} \geq 0$ is a constant depending only on $\epsilon$.

Proof. By (5.5) and (5.2),

$$
\begin{aligned}
\psi(t) & =\int_{t}^{\infty} e^{-\rho s}\left[\left[Y_{*}(t)\right]^{*}\right]^{-1}\left[Y_{*}(s)\right]^{*} \frac{\partial g\left(x_{*}(s), u_{*}(s)\right)}{\partial x} d s \\
& =\int_{t}^{\infty} e^{-\rho s}\left[Y_{*}(s)\left[Y_{*}(t)\right]^{-1}\right]^{*} \frac{\partial g\left(x_{*}(s), u_{*}(s)\right)}{\partial x} d s \\
& =\int_{t}^{\infty} e^{-\rho s}[K(s, t)]^{*} \frac{\partial g\left(x_{*}(s), u_{*}(s)\right)}{\partial x} d s,
\end{aligned}
$$

where $Y_{*}=Y_{\left(u_{*}, x_{*}\right)}$ is a normalized fundamental solution matrix of (5.10) and $K(s, t)=Y_{*}(s)\left[Y_{*}(t)\right]^{-1}$ is the Cauchy matrix of (5.10). Hence, by (A6), (A7), and 
(5.9), for any $0<\epsilon<\min \{\rho-\lambda, \rho-(r+1) \lambda\}$, we have

$$
\begin{aligned}
\|\psi(t)\| & \leq \int_{t}^{\infty} e^{-\rho s}\left\|[K(s, t)]^{*}\right\|\left\|\frac{\partial g\left(x_{*}(s), u_{*}(s)\right)}{\partial x}\right\| d s \\
& \leq C_{12} \int_{t}^{\infty} e^{-\rho s} e^{\bar{\lambda}(s-t)} e^{\epsilon s}\left(1+e^{r \lambda s}\right) d s \\
& \leq \kappa_{2}\left(e^{-\rho t+\epsilon t}+e^{-(\rho-r \lambda) t+\epsilon t}\right),
\end{aligned}
$$

where $C_{12} \geq 0$ and $\kappa_{2} \geq 0$ depend only on $\epsilon$. Hence, estimate (5.11) holds for any $\epsilon>0$.

Note that if $\rho>0$, then Corollary 6 implies the validity of both (2.1) and (2.2).

Now, let us consider the situation where the control system (1.1) is linear and stationary. Problem (P) is specified as follows.

Problem (P1):

$$
\begin{gathered}
\dot{x}(t)=F x(t)+u(t), \quad u(t) \in U ; \\
x(0)=x_{0} ; \\
\operatorname{maximize} J(x, u)=\int_{0}^{\infty} e^{-\rho t} g(x(t), u(t)) d t ;
\end{gathered}
$$

here $F$ is a real $n \times n$ matrix.

Let $\lambda_{F}$ be the maximum of the real parts of the eigenvalues of $F$. Then $\lambda_{F}$ is the maximal number from the Lyapunov spectrum of the linear homogenous differential equation corresponding to (5.12), and for any $\epsilon>0$, we have

$$
\left\|e^{F t}\right\| \leq C_{13} e^{\left(\lambda_{F}+\epsilon\right) t} \quad \text { for all } t \geq 0 .
$$

Here, $e^{F t}$ is the exponential of matrix $F$ and $C_{13} \geq 0$ is a constant depending only on $\epsilon$ (see [20]). A standard representation of a solution to (5.12) through the matrix exponential $e^{F t}$ (see [25]) implies that for any $\epsilon>0$ and for any admissible trajectory $x$ of system (5.12), it holds that

$$
\|x(t)\| \leq C_{14}+C_{15} e^{\left(\lambda_{F}+\epsilon\right) t} \quad \text { for all } t \geq 0,
$$

where $C_{14} \geq 0$ and $C_{15} \geq 0$ depend only on $\epsilon$. Thus, for an arbitrary $\lambda>\lambda_{F}$, assumption (A7) is satisfied. The latter observation and the fact that every linear stationary equation is regular [20] imply the following specification of Corollary 6 for problem (P1).

COROLlary 7. Let assumptions (A3), (A4), and (A6) be satisfied and let $\rho>$ $(r+1) \lambda_{F}$. Let $\left(u_{*}, x_{*}\right)$ be an admissible pair optimal in problem $(\mathrm{P} 1)$. Then there exists an adjoint variable $\psi$ associated with $\left(u_{*}, x_{*}\right)$ such that

(i) $\left(u_{*}, x_{*}\right)$ satisfies relations (1.7) and (1.8) of the normal-form core Pontryagin maximum principle together with $\psi$;

(ii) $\left(u_{*}, x_{*}\right)$ and $\psi$ satisfy the normal-form stationarity condition (3.18);

(iii) for every $\epsilon>0$, it holds that

$$
\|\psi(t)\| \leq \kappa_{3}\left(e^{-\rho t+\epsilon t}+e^{-\left(\rho-r \lambda_{F}\right) t+\epsilon t}\right) \quad \text { for all } \quad t \geq 0,
$$

where $\kappa_{3} \geq 0$ is a constant depending only on $\epsilon$.

Note that if $\rho>0$, then Corollary 7 implies the validity of both (2.1) and (2.2). 


\section{REFERENCES}

[1] K. Arrow, Application of control theory to economic growth, in Mathematics of the Decision Sciences, Part 2, AMS, Providence, RI, 1968, pp. 85-119.

[2] K. Arrow and M. Kurz, Public Investment, the Rate of Return, and Optimal Fiscal Policy, The Johns Hopkins University Press, Baltimore, MD, 1970.

[3] A. V. Arutyunov, Perturbations of extremal problems with constraints and necessary optimality conditions, in Mathematical Analysis, Vol. 27, Itogi Nauki i Tekhniki, VINITI, Moscow, 1989, pp. 147-235 (in Russian); J. Soviet Math., 54 (1991), pp. 1342-1400 (in English).

[4] A. V. Arutyunov and S. M. Aseev, Investigation of the degeneracy phenomenon of the maximum principle for optimal control problems with state constraints, SIAM J. Control Optim., 35 (1997), pp. 930-952.

[5] S. M. AsEev, The method of smooth approximations in the theory of necessary optimality conditions for differential inclusions, Izv. Math., 61 (1997), pp. 235-258.

[6] S. M. AsEev, Methods of regularization in nonsmooth problems of dynamic optimization, J. Math. Sci. (New York), 94 (1999), pp. 1366-1393.

[7] S. M. AsEev, Extremal problems for differential inclusions with state constraints, Proc. Steklov Inst. Math., 233 (2001), pp. 1-63.

[8] S. Aseev, G. Hutschenreiter, and A. Kryazhimskit, A Dynamic Model of Optimal Allocation of Resources to REDD, IIASA Interim Report IR-02-16, Laxenburg, Austria, 2002.

[9] S. M. Aseev, A. V. Kryazhimskit, and A. M. Tarasyev, First Order Necessary Optimality Conditions for a Class of Infinite-Horizon Optimal Control Problems, IIASA Interim Report IR-01-007, Laxenburg, Austria, 2001.

[10] S. M. Aseev, A. V. Kryazhimskit, and A. M. Tarasyev, The Pontryagin maximum principle and transversality conditions for an optimal control problem with infinite time interval, Proc. Steklov Inst. Math., 233 (2001), pp. 64-80.

[11] S. M. Aseev, A. V. Kryazhimskit, and A. M. TARAsyev, The maximum principle and transversality conditions for a class of optimal economic growth problems, in Proceedings of the 5th IFAC Symposium on Nonlinear Control Systems (St.-Petersburg, Russia), A. L. Fradkov and A. B. Kurzhanski, eds., Elsevier, New York, 2002, pp. 64-68.

[12] J. P. Aubin and F. H. Clarke, Shadow prices and duality for a class of optimal control problems, SIAM J. Control Optim., 17 (1979), pp. 567-586.

[13] E. J. BALDER, An existence result for optimal economic growth problems, J. Math. Anal. Appl., 95 (1983), pp. 195-213.

[14] R. Barro and X. Sala-I-Martin, Economic Growth, McGraw-Hill, New York, 1995.

[15] L. M. Benveniste and J. A. Scheinkman, Duality theory for dynamic optimization models of economics: The continuous time case, J. Econom. Theory, 27 (1983), pp. 1-19.

[16] J. Blot And P. Michel, First-order necessary conditions for infinite-horizon variational problems, J. Optim. Theory Appl., 88 (1996), pp. 339-364.

[17] D. A. Carlson, A. B. Haurie, and A. Leizarowitz, Infinite Horizon Optimal Control. Deterministic and Stochastic Systems, Springer-Verlag, Berlin, 1991.

[18] L. Cesari, Asymptotic Behavior and Stability Problems in Ordinary Differential Equations, Springer-Verlag, Berlin, 1959.

[19] L. Cesari, Optimization-Theory and Applications. Problems with Ordinary Differential Equations, Springer-Verlag, New York, 1983.

[20] B. P. Demidovich, Lectures on Mathematical Stability Theory, The Moscow State University Press, Moscow, 1998 (in Russian).

[21] I. Ekeland, Some variational problems arising from mathematical economics, in Mathematical Economics, Lecture Notes in Math. 1330, Springer-Verlag, Berlin, 1988, pp. 1-18.

[22] A. F. Filippov, On certain questions in the theory of optimal control, Vestn. Mosk. Univ. Ser. Mat. Mekh. Astron. Fiz. Khom., 2 (1959), pp. 25-32 (in Russian); J. Soc. Indust. Appl. Math. Ser. A: Control, 1 (1962), pp. 76-84 (in English).

[23] G. M. Grossman and E. Helpman, Innovation and Growth in the Global Economy, MIT Press, Cambridge, MA, 1991.

[24] H. Halkin, Necessary conditions for optimal control problems with infinite horizons, Econometrica, 42 (1974), pp. 267-272.

[25] P. Hartman, Ordinary Differential Equations, John Wiley and Sons, New York, 1964.

[26] T. Kaminigashi, Necessity of transversality conditions for infinite horizon problems, Econometrica, 69 (2001), pp. 995-1012.

[27] T. Koopmans, Objectives, constraints, and outcomes in optimal growth models, Econometrica, 35 (1967), pp. $1-15$

[28] S. F. LEUnG, Transversality condition and optimality in a class of infinite horizon continuous 
time economic models, J. Econom. Theory, 54 (1991), pp. 224-233.

[29] A. M. Lyapunov, Probléme général de la stabilité du mouvement, Ann. of Math. Stud. 17, Princeton University Press, Princeton, NJ, 1947.

[30] O. L. Mangasarian, Sufficient conditions for the optimal control of nonlinear systems, SIAM J. Control, 4 (1966), pp. 139-152.

[31] P. Michel, On the transversality conditions in infinite horizon optimal problems, Econometrica, 50 (1982), pp. 975-985.

[32] B. Sh. Mordukhovich, Approximation Methods in Problems of Optimization and Control, Nauka, Moscow, 1988 (in Russian).

[33] W. D. Nordhaus, Managing the Global Commons. The Economics of Climate Change, MIT Press, Cambridge, MA, 1994.

[34] L. S. Pontryagin, V. G. Boltyanskit, R. V. Gamkrelidze, and E. F. Mishchenko, The Mathematical Theory of Optimal Processes, Interscience Publishers, John Wiley and Sons, New York, 1962.

[35] F. P. Ramsey, A mathematical theory of saving, Econom. J., 38 (1928), pp. 543-559.

[36] A. Seierstad And K. Sydseter, Optimal Control Theory with Economic Applications, NorthHolland, Amsterdam, 1987.

[37] K. Shell, Applications of Pontryagin's maximum principle to economics, in Mathematical Systems Theory and Economics I, H. W. Kuhn and G. P. Szegö, eds., Springer-Verlag, Berlin, 1969, pp. 241-292.

[38] G. V. Smirnov, Transversality conditions for infinite horizon problems, J. Optim. Theory Appl., 88 (1996), pp. 671-688.

[39] R. M. Solow, Growth Theory: An Exposition, Oxford University Press, New York, 1970. 


\section{Additional copies}

Further copies of this IIASA Reprint are available online at www.iiasa.ac.at/Publications

Hard copies are also available for a small handling charge. Orders must include the publication number and should be sent to the Publications Department, International Institute for Applied Systems Analysis, A-2361 Laxenburg, Austria.

Telephone: +432236807

Telefax: +432236 71313

E-mail: publications@iiasa.ac.at 
International Institute for Applied Systems Analysis

Schlossplatz 1, A-2361 Laxenburg, Austria

Tel: +432236807 Fax: +43223671313

I I AS A www.iiasa.ac.at 